\title{
Chiral Thermodynamics in Tailored Chiral Optical Environments
}

\author{
Gabriel Schnoering $\odot^{*}$ Samuel Albert $\odot$, Antoine Canaguier-Durand, ${ }^{\dagger}$ and Cyriaque Genet $\odot^{*}$ \\ Université de Strasbourg, CNRS, Institut de Science et d'Ingénierie Supramoléculaires, \\ UMR 7006, F-67000 Strasbourg, France
}

(Received 21 December 2020; revised 30 July 2021; accepted 27 August 2021; published 1 November 2021)

\begin{abstract}
The stochastic motion of Brownian particles out of equilibrium yields rich thermodynamic landscapes studied on a great variety of systems through many different research fields. Here, we study within the field of stochastic thermodynamics the dynamics and energetics of an overdamped Brownian chiral nanoparticle diffusing in a symmetric bistable optical potential formed in the standing wave of two counterpropagating Gaussian beams. Control on the polarizations of each beam creates chiral optical environments by endowing the standing wave with optical chiral densities or optical chiral fluxes without modifying the initial bistability. These chiral densities and fluxes are associated, respectively, with reactive or dissipative chiral optical forces exerted on the diffusing chiral nanoparticle. This optomechanical chiral coupling leads to a modification of the thermal activation process in ways that depend on the nanoparticle enantiomer and on the enantiomorphism of the optical field. Reactive chiral forces contribute to a global enantiospecific change of the Helmholtz free energy, but preserving the symmetry of the bistable potential. Dissipative chiral forces correspond to a nonequilibrium steady state where the barrier-crossing rates become asymmetric while leaving unaffected the initial potential. This symmetry breaking is associated with heat transferred to the thermal bath that can be evaluated. The symmetry breaking yields chiral deracemization schemes that can be explicitly calculated and simulated. Our results reveal how chiral degrees of freedom of both the nanoparticle and the optical field transform into true thermodynamic control parameters. The resulting optomechanical model gives way to new opportunities in the context of chiral sensing at the single-nanoparticle level and to original strategies for chiral discrimination at the nanoscale using the observables associated with the thermodynamics at play, such as escape rates or probability density functions.
\end{abstract}

DOI: $10.1103 /$ PhysRevX.11.041022

Subject Areas: Optics, Statistical Physics

\section{INTRODUCTION}

When a chiral system is immersed within a chiral environment, specific interactions can be induced that combine the chirality of both the system and the environment. This is known as chiral coupling and leads to a great variety of manifestations and effects. For instance, the trajectories of spins in magnetic fields are determined by a chiral coupling exploited in Stern-Gerlach interferometry [1]. Also, chiral coupling is at play in unidirectional

\footnotetext{
*Present address: Laboratory of Thermodynamics in Emerging Technologies, ETH Zürich, Sonneggstrasse 3, CH-8092 Zürich, Switzerland.

${ }^{\dagger}$ Present address: Saint-Gobain Research Paris, 39 quai Lucien Lefranc, F-93300 Aubervilliers, France.

${ }^{\ddagger}$ genet@unistra.fr

Published by the American Physical Society under the terms of the Creative Commons Attribution 4.0 International license. Further distribution of this work must maintain attribution to the author(s) and the published article's title, journal citation, and DOI.
}

molecular motions induced inside ratchet potentials, in so-called molecular machine configurations [2,3].

Chiral coupling naturally depends on the chirality of the system, a property known as enantiodependence, which is central to the field of analytical chemistry. There, the notion of asymmetric chemical evolution within chiral environments permeates vast literature covering a wide range of topics [4-6]. Chiral-liquid-crystals nuclear magnetic resonance (NMR) and chiral chromatography both exploit the fact that in chiral solvents, solute-solvent interactions are enantiodependent. In NMR, these interactions lead to differential orientations of molecular enantiomers with respect to the magnetic field. As a consequence, NMR parameters, such as residual chemical shift anisotropy, as well as residual dipolar or quadrupolar coupling, are enantiomerically dependent, achieving high-resolution chiral discrimination capacities [7-9]. In chiral chromatography too, the chirality of the stationary phase (the chiral selector) is crucial for forming, through noncovalent interactions between the enantiomers and the chiral selector, diastereoisomer complexes that have different free energies depending on the enantiomer. Differences in free 
energies lead to driving forces for retention in the column that become enantiodependent $[10,11]$.

Chiral coupling can also yield enantioselective effects. Among many possible early anticipated-notably by Le Bel and Van't Hoff in the 19th century-Kagan et al. have reported that an asymmetric synthesis can be triggered when irradiating the reactants with circularly polarized light [12]. In this asymmetric chemistry, circularly polarized light acts as a reactive in the synthesis to lead to enantiomeric excess in the product formation [13,14]. Forming enantiomerically pure phases while starting from mixtures containing even quantities of left- vs right-handed chiral molecules, i.e., so-called racemates, is a process known as deracemization of paramount importance in chemistry and for the pharmaceutical industry. Deracemization processes also torment the search for the origin of homochirality [15-18]. They can result from spontaneous mirror symmetry-breaking effects when an initial stochastic selection of one enantiomer followed by an amplification or autocatalytic process can lead to the full predominance of only one chirality, being dextro for sugars or levo for amino acids [19,20]. They can also result from some systematic coupling mechanisms that can lead to the asymmetry observed in biochemistry [21]. Such coupling mechanisms can rely on fundamental asymmetries, such as the violation of parity $[22,23]$ or can rely on the role of the environment (be it electromagnetic, chemical, geological, astronomical, and so forth) whose chirality determines the direction of the splitting in free energies [24].

In all these examples, the thermodynamics involved in the variety of deracemization processes (by crystallization, chemistry, light, etc.) is not easy to resolve, and the precise role played by the chiral coupling engaged in each process is not easy to uncover. In this work, we address head-on the thermodynamic question by looking at one particular type of chiral coupling induced when a chiral scattering object is immersed within a chiral electromagnetic field. This chiral coupling manifests itself by the emergence of chiral optical forces that have been recently discovered [25-28]. Because they intertwine the chiral content of the electromagnetic field with the chiral response of the object, the new forces are enantioselective and have led to promising all-optical chiral sorting strategies [29-35]. These strategies have obviously a strong applicative potential at the nanoscale when targeting molecular chiral resolution by optomechanical means [36].

At these scales, thermal fluctuations impose a stochastic description of the action of the chiral forces on the chiral nano-object behaving as a Brownian system. Capable of knowing exactly the force fields at play, the purpose of our work is to determine the thermodynamic consequences of the asymmetric evolution of our Brownian system under the influence of the optomechanical chiral coupling. As we show, the methods of stochastic thermodynamics [37-40] are remarkably appropriate for shaping a comprehensive view on the thermodynamics of this optomechanical chiral

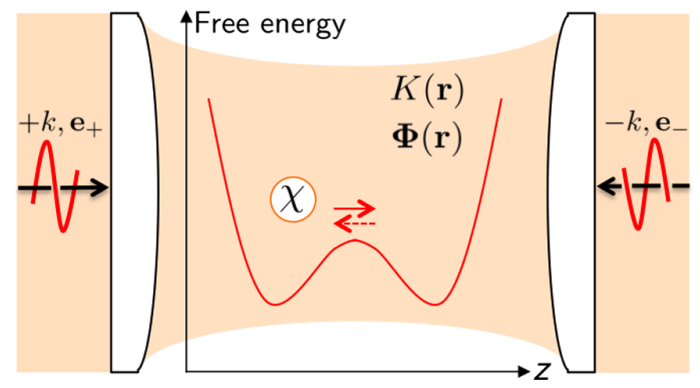

FIG. 1. Schematics of the proposed optomechanical model. Two counterpropagating $\pm k$ Gaussian beams focused on a common waist by two objectives create a bistable potential free-energy surface. A diffusing chiral nanoparticle optically trapped in this potential in the dipolar regime is thermally activated and crosses, in both $z \lessgtr 0$ directions, the barrier separating the two potential wells. Depending on the settings of the polarization vectors $\mathbf{e}_{ \pm}$of the beams, optical chiral density $K(\mathbf{r})$ and/or chiral flux $\boldsymbol{\Phi}(\mathbf{r})$ can be induced in the standing wave between the objectives. When this happens, a chiral coupling involves the chirality of the nanoparticle via the chiral polarizability $\chi$ and the chirality of the field via $K(\mathbf{r})$ and $\boldsymbol{\Phi}(\mathbf{r})$, which are, respectively, a time-even pseudoscalar and pseudovector, i.e., truly chiral quantities [46]. The chiral coupling generates chiral optical forces that act on the nanoparticle and bias the diffusing motion of the nanoparticle within the bistable potential. These forces therefore endow the thermally activated barrier crossing with an enantiospecific, chiral discriminative, thermodynamics.

coupling and for deriving important results related to chiral asymmetric evolutions.

To do so, we build the three-dimensional (3D) chiral optomechanical model schematized in Fig. 1. By giving the optical environment - that shapes the trapping potentialenantiomorphic features fixed independently from the enantiomeric form of the Brownian object, we demonstrate how the chirality of both the object and the environment determines the coupling and, in turn, fixes the stochastic energetics of the Brownian dynamics performed within an intrinsically chiral 3D free-energy landscape. We set our model in the (archetypical) Kramers framework for its capacity to describe thermodynamic processes (thermal activation of a barrier crossing) at the heart of diffusion models of chemical reactions [41,42]. The so-called Kramers problem is therefore immediately connected with the field of chiral chemistry where a great variety of chiral molecular systems do exhibit thermally activated bistability $[43,44]$. The bistable potential is indeed, and even before Kramers, central to the first historical explanation of the stability of chiral molecules in the so-called Hund's paradox [45]. Room-temperature fast interconversions of enantiomers through conformational barriers are usually modeled as dynamical processes that lead, in the majority of cases, to racemic solutions. In this context, bistability provides a conceptual framework for describing such interconversions. But as we demonstrate, it also allows us to investigate the influence of the chiral coupling on the 
thermodynamics of the barrier crossing manifested in modifications of the interconversion rates that favor one enantiomer with respect to the other.

With this model and through the methods of stochastic thermodynamics, we are able to describe explicitly the thermodynamic consequences of the chiral coupling. We show how the energetics and the barrier crossings depend not only on the enantiomeric form of the chiral particle but on the enantiomorphism of the chiral optical environment too. We describe the emergence of an enantiospecific thermodynamics where the chiral coupling transforms chiral (internal) degrees of freedom of the Brownian system and of the light field into true thermodynamic control parameters. This transformation constitutes a central outcome of our work.

We also show how different thermodynamic landscapes are set by the different (reactive vs dissipative) natures of the chiral optical environment that can be selected through a careful engineering of the optical environment. This engineering, in particular, leads us to measure the actual thermodynamic cost of a deracemization process induced within our framework. Echoing Pasteur's views of "asymmetric forces" capable of lifting the enantiomeric free-energy degeneracy [47], our approach provides a concrete framework for investigating further fundamental issues related to chiral selection and their thermodynamic consequences. It also provides the right settings to envision future experiments nurtured by theory in an exchange that has fertilized to date this newly born field of chiral optomechanics.

\section{SUMMARY OF OUR FRAMEWORK}

From a dynamical viewpoint, the coupling between a chiral dipole and a chiral electromagnetic field (for instance, a circularly polarized light field) leads to specific types of optical forces, chiral in nature, that are exerted on the chiral dipole, as already discussed in Ref. [25]. Their general structure, in particular, their reactive (conservative) and dissipative (nonconservative) nature, central to this work, is reviewed in Sec. III of the manuscript. We explain the coupling mechanism through which new chiral optical forces emerge as Pasteurian forces from the "immersion" of the chiral dipole within a chiral electromagnetic environment. For the reasons exposed in the Introduction, we set this optical chiral coupling in a specific dual-beam optical configuration designed in such a way as to form a bistable optical trapping potential capable of implementing a Brownian thermal activation process. The dual-beam configuration and the associated double-well potential energy with its barrier height profile are detailed in Sec. IV.

Our concept of tailored chiral optical environments is presented in Sec. V where we show that, keeping the electromagnetic energy density fixed, a fine polarization control enables us to induce chiral densities and fluxes within the dual-beam interfering pattern in ratios fully controllable by the polarization parameters. The key feature here is the possibility to choose these polarization settings without modifying at all the bistable structure of the achiral potential. As we show below, this possibility stems from the fact that the bistability and the chiral optical densities and fluxes are determined by different parts of the electromagnetic energy density and can thus be fixed independently from each other.

In this section, different types of chiral optical environments are generated depending on the selection of either pseudo-scalar chiral densities or pseudo-vector chiral fluxes. Through the chiral-coupling mechanism, the nature of the environment then leads to the induction of specific chiral optical forces: conservative for chiral optical densities, or nonconservative for chiral optical fluxes. In other words, conservative and/or nonconservative chiral forces can be selected simply by tuning the relative helicities of both beams. These one-to-one relations explain why we speak of reactive or dissipative chiral optical environments. As one important piece of our work, we emphasize that in the conservative case, the chiral coupling defines a pseudoscalar potential with a truly chiral nature following Barron's classification [46].

Section VI builds the stochastic model of a Brownian chiral nanoparticle optically trapped within a bistable potential energy in order to evaluate the thermodynamics consequences of the chiral coupling in both reactive (Sec. VI A) and dissipative (Sec. VI B) cases. This section presents a Fokker-Planck description involving the probability current along the optical axis that we use in order to calculate escape rates from both sides of the potential barrier.

In the stochastic thermodynamic framework of the Kramers problem [41,48], we calculate in Sec. VI A the modification of the escape rates induced by immersing the chiral nanoparticle inside a reactive chiral environment. Induced chiral forces being conservative contribute to a global change of the Helmholtz free-energy landscape yielding new potential energy surfaces that depend both on the dipole enantiomer and the optical field enantiomorph. These new potential energy surfaces remain symmetric, with a preserved degeneracy in the barrier-crossing rates. In contrast, when the chiral coupling is set to be dissipative in Sec. VI B, the chiral optical forces exerted on the chiral dipole are nonconservative, and the mechanical energy is transferred by the chiral optical field to the dipole that is dissipated as heat into the thermal bath. In this nonequilibrium steady-state configuration, the probability density function associated with the barrier-crossing diffusion is modified but not by a change in the Helmholtz free energy as in the reactive case. More precisely, the nonconservative chiral forces break the symmetry of the probability density function while leaving the potential unaffected. This symmetry breaking is one important result of the work since it corresponds exactly to a chiral separation process. It is accompanied by the creation of 
heat, thus entropy, that can be interpreted as the thermodynamic cost for assigning enantiomers to specific wells of the bistable optical potential, in analogy with the so-called entropy penalty of deracemization with respect to racemization $[49,50]$.

The relevance of the Kramers framework for extracting the thermodynamics significance of our chiral optomechanical model is confirmed in Sec. VII by three-dimensional stochastic simulations of an overdamped Langevin equation. The Langevin approach gives access to the individual Brownian trajectory for the diffusion of a chiral nanosphere inside the bistable optical trap in the presence of either reactive or/and dissipative chiral optical forces. We then simulate the diffusive motions of a large number of chiral nanospheres to build a statistical ensemble that greatly helps in visualizing the mechanical action of the chiral optical environment when calculating the probability density functions associated with the ensemble of trajectories. Consolidating our model approach based on escape rates, the simulations highlight the chiral thermodynamic deracemization scheme enabled by the dissipative chiral coupling as a possible chiral resolution strategy.

The results are complemented in Sec. VIII with statistics performed on the barrier-crossing events at the level of single trajectories. These statistics lead to precise determinations of the average residency times in each of the bistable local wells. They actually point to the possibility to measure the chiral optical forces biasing the thermodynamics of the barrier crossing by recording only residency times. Exchanging thereby force measurements into time measurements paves the way to shortcut force calibration procedures and as such, to a new strategy interesting to develop in the context of weak chiral force measurements. In particular, obtaining the average residency times in both the reactive and dissipative chiral-coupling schemes yields an absolute measurement of both real and imaginary parts of the chiral polarizability of a single nanoparticle.

\section{A REMINDER ON OPTOMECHANICAL CHIRAL COUPLING: ACHIRAL AND CHIRAL OPTICAL FORCES}

We review here the general expression of the timeaveraged optical force $\mathbf{F}(\mathbf{r})$ exerted by a harmonic electromagnetic complex field $\left[\mathbf{E}(\mathbf{r}) e^{-i \omega t}, \mathbf{H}(\mathbf{r}) e^{-i \omega t}\right]$ on a chiral nanoparticle modeled by a chiral dipole - not to be confused with a mere anisotropic dipole-characterized by electric $\mathbf{p}$ and magnetic $\mathbf{m}$ dipolar moments coupled to the incident electric and magnetic fields through complex electric $\alpha$, magnetic $\beta$, and mixed electric magnetic $\chi$ polarizabilities as $[25,51]$

$$
\left(\begin{array}{c}
\mathbf{p} \\
\mathbf{m}
\end{array}\right)=\left(\begin{array}{cc}
\alpha \varepsilon_{f} & i \chi \sqrt{\varepsilon_{f} \mu_{f}} \\
-i \chi \sqrt{\varepsilon_{f} / \mu_{f}} & \beta
\end{array}\right)\left(\begin{array}{l}
\mathbf{E} \\
\mathbf{H}
\end{array}\right),
$$

where $\varepsilon_{f}, \mu_{f}$ are the permittivity and permeability of the fluid enclosed in the optical trapping cell (deionized water).

As now well known, the time-averaged optical force $\mathbf{F}(\mathbf{r})$ splits into (standard) achiral and (new) chiral contributions. We defer to the Appendix A the full expression for $\mathbf{F}(\mathbf{r})$ and neglect here and below all magnetic force contributions. This allows us to write

$$
\begin{aligned}
\mathbf{F}(\mathbf{r}) & =\operatorname{Re}\left[\alpha \mathbf{f}_{0}(\mathbf{r})+\frac{\chi}{\omega \sqrt{\varepsilon_{f} \mu_{f}}} \mathbf{h}_{0}(\mathbf{r})\right] \\
& =\mathbf{F}_{\alpha}(\mathbf{r})+\mathbf{F}_{\chi}(\mathbf{r}),
\end{aligned}
$$

where $\mathbf{F}_{\alpha}(\mathbf{r})$ is the standard achiral optical force contribution that involves only $\alpha$, and $\mathbf{F}_{\chi}(\mathbf{r})$ is the new chiral optical force contribution that depends on the mixed electric magnetic $\chi$ polarizability. Both achiral and chiral force contributions can be separated into reactive and dissipative components [25,52] engaging, respectively, the real and imaginary parts (i) of the $(\alpha, \chi)$ polarizabilities and (ii) of the vector fields $\left(\mathbf{f}_{0}, \mathbf{h}_{0}\right)$ that take simple forms with

$$
\begin{gathered}
\operatorname{Re}\left[\mathbf{f}_{0}(\mathbf{r})\right]=\nabla W_{E}(\mathbf{r}), \\
\operatorname{Im}\left[\mathbf{f}_{0}(\mathbf{r})\right]=-\omega \varepsilon_{f} \mu_{f} \boldsymbol{\Pi}_{0}(\mathbf{r}), \\
\operatorname{Re}\left[\mathbf{h}_{0}(\mathbf{r})\right]=\nabla K(\mathbf{r}), \\
\operatorname{Im}\left[\mathbf{h}_{0}(\mathbf{r})\right]=-2 \omega \varepsilon_{f} \mu_{f}[\boldsymbol{\Phi}(\mathbf{r})-\nabla \times \boldsymbol{\Pi}(\mathbf{r}) / 2] .
\end{gathered}
$$

The achiral contribution therefore is determined by $W_{E}(\mathbf{r})=\varepsilon_{f} \mathbf{E}(\mathbf{r}) \cdot \mathbf{E}^{*}(\mathbf{r}) / 4$ the time-averaged (electric) energy density and $\boldsymbol{\Pi}_{0}(\mathbf{r})$ the orbital part of the full Poynting vector $\Pi(\mathbf{r})=\operatorname{Re}\left[\mathbf{E}(\mathbf{r}) \times \mathbf{H}^{*}(\mathbf{r})\right] / 2$ showing how the reactive achiral force component can be interpreted as a gradient force and the dissipative one as a radiation pressure, as already discussed in Refs. [53,54]. For the chiral contribution, the chiral density $K(\mathbf{r})=\omega \varepsilon_{f} \mu_{f} \operatorname{Im}[\mathbf{E}(\mathbf{r})$. $\left.\mathbf{H}^{*}(\mathbf{r})\right] / 2$ and the chiral flux $\boldsymbol{\Phi}(\mathbf{r})=-\omega \operatorname{Im}\left[\varepsilon_{f} \mathbf{E}(\mathbf{r}) \times \mathbf{E}^{*}(\mathbf{r})+\right.$ $\left.\mu_{f} \mathbf{H}(\mathbf{r}) \times \mathbf{H}^{*}(\mathbf{r})\right] / 4$ measure the chirality of the electromagnetic field. Here, we stress that $K(\mathbf{r})$ and $\boldsymbol{\Phi}(\mathbf{r})$ are time-even parity-odd quantities, therefore truly chiral according to Barron's definition [46].

These remarkable expressions reveal new types of optical forces, chiral in nature, that are induced when a chiral system is immersed within an electromagnetic field that contains either nonzero electromagnetic chiral density or chiral flux. These chiral optical forces can therefore be viewed as the dynamic manifestation of a chiral coupling between light and matter. They intertwine the chirality of the matter with the chirality of the electromagnetic field and are enantioselective, explaining why they generated a strong interest since their predictions. Dipolar, they also do not depend on any specific energy-level structure of the chiral system involved. However, essentially because $\chi \ll \alpha$, chiral optical forces 
remain small compared to achiral optical forces. This issue has driven many proposals for exploiting the potential of these chiral optical forces in chiral discriminatory schemes despite the fact that they correspond to relatively weak signals $[25,27,29,30,32-35,55]$.

The chiral light-matter coupling leads to simple relations. From the light part, chiral electromagnetic fields form a pair of enantiomorph optical environments when reversing the signs of $K(\mathbf{r}), \boldsymbol{\Phi}(\mathbf{r})$ without changing the energy density. From the matter part, chiral dipoles form a pair of enantiomers with opposite signs for the real and imaginary parts of $\chi$. We decide here to call a "right-handed" dipole one with $\operatorname{Re}[\chi]>0, \operatorname{Im}[\chi]<0$ and a "left-handed" dipole with $\operatorname{Re}[\chi]<0, \operatorname{Im}[\chi]>0$. In the model presented below, we fix a ratio $\chi / \alpha=5 \%$ calculated from the ClausiusMossotti polarizabilities $\alpha$ and $\chi$ in the quasistatic limit; see Appendix B for details.

\section{BISTABLE POTENTIAL ENERGY IN AN OPTICAL TRAP}

The expressions of the optical forces being reminded, we now explain how the achiral force contribution can induce a bistable dynamics within the optical trap. To do so, i.e., to form a double-well trapping potential, we use a trapping configuration involving two counterpropagating Gaussian beams focused on a common waist already implemented in the context of optical force spectroscopy [56-58]. In the paraxial approximation [59] and using harmonic-timedependent complex fields, the Gaussian beams propagating either with $\mathrm{a}+k z$ or $-k z$ phase along the $z$-optical axis $\left(k=\sqrt{\varepsilon_{f}} \omega / c\right)$ can be evaluated at any position $\mathbf{r}=q \hat{\boldsymbol{\rho}}+$ $z \hat{\mathbf{z}}$ in the cylindrical coordinate system as

$$
\begin{gathered}
\mathbf{E}_{ \pm}(\mathbf{r})=\mathcal{E}_{ \pm}(\mathbf{r}) e^{ \pm i k z} \mathbf{e}_{ \pm}, \\
\mathbf{H}_{ \pm}(\mathbf{r})=\frac{1}{Z_{f}} \mathcal{E}_{ \pm}(\mathbf{r}) e^{ \pm i k z}\left( \pm \hat{\mathbf{z}} \times \mathbf{e}_{ \pm}\right),
\end{gathered}
$$

where $\mathbf{e}_{ \pm}$are the (unit) polarization vectors associated with each field in each direction of propagation and $Z_{f}=$ $\sqrt{\mu_{f} / \varepsilon_{f}}$ the optical impedance of the fluid. With beam waists $w_{0}$ and Rayleigh ranges $z_{R}$ identical for both beams, we have

$$
\mathcal{E}_{ \pm}(\mathbf{r})=\mathcal{E}_{0} \frac{w_{0}}{w(z)} e^{ \pm i \phi(q, z)} e^{-\left[q^{2} / w^{2}(z)\right]}
$$

where we note $\phi(\mathbf{r})=k q^{2} / 2 R(z)-\xi(z)$ the Gaussian phase that accounts for the finite radius of curvature $R(z)=z\left[1+\left(z_{R} / z\right)^{2}\right]$ of the beam and the Gouy phase $\xi(z)=\arctan \left[z / z_{R}\right]$, and $w(z)=w_{0}\left[1+\left(z / z_{R}\right)^{2}\right]^{1 / 2}$ the beam radius measured along the optical axis from both sides of the waist. We define the polarization vectors by

$$
\begin{aligned}
& \mathbf{e}_{+}=\left(\sqrt{1-h_{+}} \mathbf{e}_{l}+\sqrt{1+h_{+}} \mathbf{e}_{r}\right) / \sqrt{2}, \\
& \mathbf{e}_{-}=\left(\sqrt{1-h_{-}} e^{i(\delta-\delta \theta)} \mathbf{e}_{l}+\sqrt{1+h_{-}} e^{i(\delta+\delta \theta)} \mathbf{e}_{r}\right) / \sqrt{2}
\end{aligned}
$$

in the basis of left and right circular polarization vectors $\mathbf{e}_{l}$ and $\mathbf{e}_{r}$, with $h_{+}$and $h_{-}$corresponding to the helicity of both beams ranging from 1 for a right-handed circular polarization to -1 for a left-handed circular polarization. The phase delay between both beams is $\delta$, and $\delta \theta$ is the angle between the semimajor axes of the polarization of both beams, as described in Fig. 2. The field superpositions $\mathbf{E}_{\mathrm{SW}}(\mathbf{r})=\mathbf{E}_{+}(\mathbf{r})+\mathbf{E}_{-}(\mathbf{r})$ and $\mathbf{H}_{\mathrm{SW}}(\mathbf{r})$ form a standing wave. A crucial consequence for the forces is the zero Poynting vector inside the standing wave because $\mathbf{E}_{\mathrm{SW}}(\mathbf{r}) \times \mathbf{H}_{\mathrm{SW}}^{*}(\mathbf{r})$ is purely imaginary.

Before inducing any chiral coupling, let us look at the dynamical landscape within the optical trap when solely involving the achiral reactive force field $\mathbf{F}_{\alpha}(\mathbf{r})=$ $\operatorname{Re}[\alpha] \nabla W_{E}(\mathbf{r})$. This force is conservative, and the corresponding potential energy inside the optical trap $U_{\text {opt }}(\mathbf{r})=$ $-\operatorname{Re}[\alpha] W_{E}(\mathbf{r})$ is determined by the time-averaged electric energy density $W_{E}(\mathbf{r})=\varepsilon_{f} \mathbf{E}_{\mathrm{SW}}(\mathbf{r}) \cdot \mathbf{E}_{\mathrm{SW}}^{*}(\mathbf{r}) / 4$ inside the standing wave.

The notations

$$
\begin{aligned}
h_{1} & =\left(\sqrt{1-h_{+}} \sqrt{1-h_{-}}-\sqrt{1+h_{+}} \sqrt{1+h_{-}}\right) / 2, \\
h_{2} & =\left(\sqrt{1-h_{+}} \sqrt{1-h_{-}}+\sqrt{1+h_{+}} \sqrt{1+h_{-}}\right) / 2, \\
\varphi(\mathbf{r}) & =\delta+2 k\left(z+\frac{q^{2}}{2 R(z)}\right)-2 \xi(z)
\end{aligned}
$$
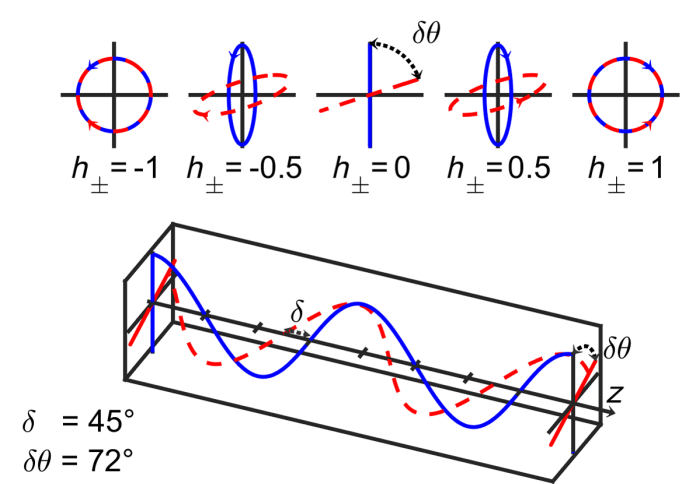

FIG. 2. The polarization vectors $\mathbf{e}_{ \pm}$for each of the two counterpropagating Gaussian beams are represented for planewave electric fields. In blue, a beam with $\mathbf{e}_{+}$and $k>0$ and in red, with $\mathbf{e}_{-}$and $k<0$. The schematics illustrates the effects of the phase $\delta$ and the polarization main axis angle $\delta \theta$ on beams linearly polarized with $h_{ \pm}=0$. The insets show the polarization ellipses for different values of $h_{ \pm}$. The effect of the $\delta \theta$ parameter is seen when the beams are not circularly polarized. 
allow us to express in a simple way the separation of the energy density $W_{E}(\mathbf{r})$ between a trapping energy density $W_{\text {trap }}(\mathbf{r})$ independent of polarization and an interference energy density $W_{\text {inter }}(\mathbf{r})$ according to

$$
\begin{gathered}
W_{E}(\mathbf{r})=W_{\text {trap }}(\mathbf{r})+W_{\text {inter }}(\mathbf{r}), \\
W_{\text {trap }}(\mathbf{r})=\frac{\mathcal{E}_{0}^{2} w_{0}^{2} \varepsilon_{f}}{2 w^{2}(z)} e^{-\frac{2 q^{2}}{w^{2}(z)}},
\end{gathered}
$$
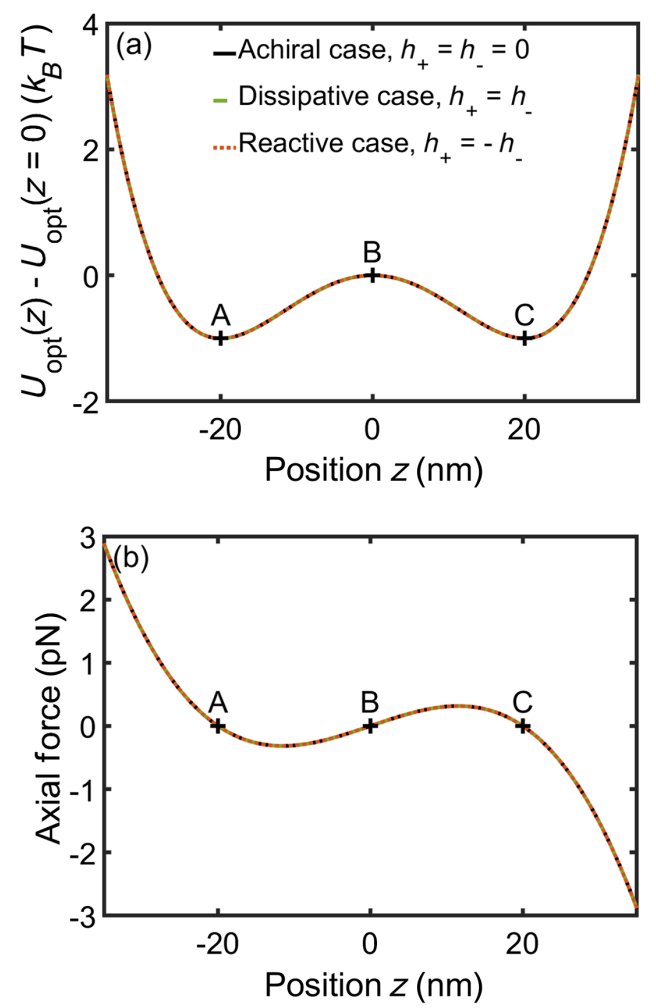

FIG. 3. (a) Bistable optical potential energy $U_{\text {opt }}(\mathbf{r})=$ $-\operatorname{Re}[\alpha] W_{E}(\mathbf{r})$ displaying the two local minima at $z_{A}$ and $z_{C}$ separated by the barrier at $z_{B}$. The electric dipolar polarizability $\alpha$ associated with an achiral $(\chi=0)$ gold $(\mathrm{Au})$ nanosphere of radius $R=20 \mathrm{~nm}$ is calculated using Au tabulated optical data measured at an illumination wavelength of $785 \mathrm{~nm}$ and the ClausiusMossotti relations of Appendix B with $\kappa_{\mathrm{Au}} \equiv 0$ in this case. (b) Corresponding optical achiral force field $\mathbf{F}_{\alpha}=\operatorname{Re}[\alpha] \nabla W_{E}(\mathbf{r})$ drawn in the waist region. This double-well profile in $U_{\text {opt }}(\mathbf{r})$ is generated with the polarization settings $h_{+}=h_{-}=0, \delta \theta=$ $0.9989 \times \pi / 2, \delta=-\pi$. Superimposed in (a) and (b) is the achiral potential energy recalculated with the polarization settings that lead to reactive and dissipative chiral environments. These settings are $h_{+}=0.05=-h_{-}, \delta \theta=0.8990 \times \pi / 2, \delta=-\pi$ for the reactive case and $h_{+}=0.0017=h_{-}, \delta \theta=\pi / 2, \delta=\pi / 2$ for the dissipative one. As seen, these polarization settings do not modify the achiral potential energy surface inside the optical trap. The distance between the two local wells located at $z_{A}<0$ and $z_{C}>0$ from both sides of the waist barrier positioned at $z_{B}=0$ is noted $\Delta \ell$ in the main text.

$$
\begin{aligned}
W_{\text {inter }}(\mathbf{r})= & W_{\text {trap }}(\mathbf{r})\left[h_{2} \cos \delta \theta \cos \varphi(\mathbf{r})\right. \\
& \left.+h_{1} \sin \delta \theta \sin \varphi(\mathbf{r})\right] .
\end{aligned}
$$

There is clearly a vast $\left(h_{1}, h_{2}, \delta, \delta \theta\right)$ parameter space available for the design of the potential energy landscape, as we discuss in detail in Appendix A. In order to set the double-well trapping potential, we start with linear polarizations $h_{+}=h_{-}=0$ giving $h_{1}=0, h_{2}=1$. The bistability profile can then be shaped by controlling the strength of the interferences superimposed to the trapping potential. This is done by adjusting $\delta \theta$ to a value that leaves only one interference falling inside the trapping envelop strong enough to cause a force inversion around the waist; see Appendix $\mathrm{C}$ for a detailed description of the landscape. This parameter being fixed, we force with $\delta$ the potential energy $U_{\text {opt }}(\mathbf{r})$ to be symmetric with respect to the waist position with a constructive interference at $z=0$. Finally, the barrier height is adjusted via the two beam (even) intensities. These controls lead to the bistable optical potential inside the trap displayed in Figs. 3(a) and 3(b) for the corresponding $z$-axial force field, with the corresponding values given in the figure caption.

\section{BISTABILITY IN CHIRAL OPTICAL ENVIRONMENTS}

The explicit expressions of the electromagnetic chiral density and chiral flux associated with the dual-beam configuration described above

$$
\begin{gathered}
K(\mathbf{r})=-\left(h_{+}-h_{-}\right) \times \omega \sqrt{\varepsilon_{f} \mu_{f}} W_{\text {trap }}(\mathbf{r}), \\
\boldsymbol{\Phi}(\mathbf{r})=-\left(h_{+}+h_{-}\right) \times \omega W_{\text {trap }}(\mathbf{r}) \hat{\mathbf{z}}
\end{gathered}
$$

immediately reveal that setting linear $h_{+}=h_{-}=0$ polarizations for both beams deprives the interference pattern from any chirality. But elliptically polarized beams endow the optical environment with chirality. This leads to the dynamical consequences that we now discuss.

The first key feature of our model is the possibility to choose $h_{+}, h_{-}$values that select $K(\mathbf{r})$ or $\boldsymbol{\Phi}(\mathbf{r})$ (or both) while preserving exactly the bistable structure of the achiral potential energy defined in Sec. IV above. This is clearly seen in Figs. 3(a) and 3(b). With such polarization choices, the double-well landscape of the trap therefore becomes optically chiral. According to Eq. (2), as soon as a chiral dipole is immersed in this chiral optical environment, the chiral coupling will induce chiral forces that add to the bistable dynamic which is, for its part, driven by the achiral force fields only.

The second important feature is the ability to select by polarization the reactive and/or dissipative nature of the chiral environment and thereby induce on the chiral dipole reactive (i.e., conservative) and dissipative (i.e., nonconservative) forces 


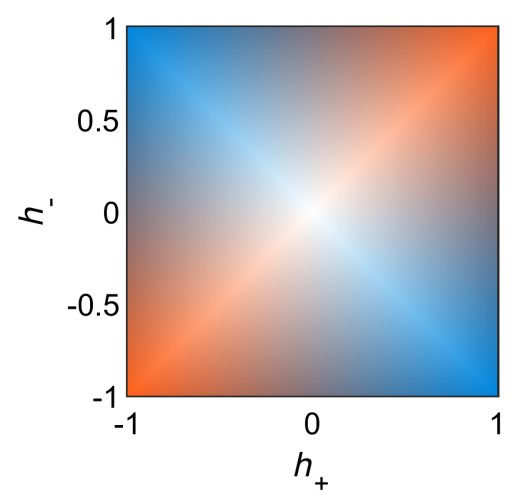

FIG. 4. Surface plot of the evolution of the reactive vs dissipative nature of the chiral optical environment in the helicity plane $\left(h_{+}, h_{-}\right)$of the two counterpropagating beams. In agreement with Eqs. (13) and (14), $c=\left|h_{+}-h_{-}\right| / 2 \times\left(c_{R}-c_{A}\right)+$ $\left|h_{+}+h_{-}\right| / 2 \times\left(c_{D}-c_{A}\right)+c_{A}$, where $c, c_{R}, c_{D}$, and $c_{A}$ are, respectively, the displayed, blue (for reactive), red (for dissipative), and white (for achiral) colors.

$$
\begin{gathered}
\mathbf{F}_{\chi}^{\mathrm{reac}}(\mathbf{r})=\operatorname{Re}[\chi] \frac{1}{\omega \sqrt{\varepsilon_{f} \mu_{f}}} \nabla K(\mathbf{r}), \\
\mathbf{F}_{\chi}^{\mathrm{diss}}(\mathbf{r})=\operatorname{Im}[\chi] 2 \sqrt{\varepsilon_{f} \mu_{f}} \mathbf{\Phi}(\mathbf{r})
\end{gathered}
$$

that are each associated with one unique chiral quantity. The evolution of the reactive vs dissipative nature of the chiral environment in the $\left(h_{+}, h_{-}\right)$helicity space is displayed in Fig. 4 where it is clear that the two distinct reactive $[K(\mathbf{r}) \neq 0, \boldsymbol{\Phi}(\mathbf{r})=\mathbf{0}]$ vs dissipative $[K(\mathbf{r})=0$, $\boldsymbol{\Phi}(\mathbf{r}) \neq \mathbf{0}]$ chiral optical environments can be selected using $h_{+}=-h_{-}$vs $h_{+}=h_{-}$. We stress that this selection is performed on a sole polarization control, without changing the intensity of the two beams. This polarization-based tailoring of the chiral optical environment yields the important thermodynamics consequences at the heart of our work.

The possibility to induce nonconservative chiral optical forces within a dissipative chiral optical environment clearly distinguishes our work from previously studied chiral-coupling configurations such as spins in magnetic fields and ratchet dynamics in molecular motor systems. This difference is due to the fact that in the latter systems, all forces derive from a potential, while in our dissipative chiral-coupling case, the nonconservative nature of the force forbids the definition of such a potential [60].

Importantly, the appropriate choices of polarizations that induce chirality without perturbing the achiral bistable potential energy set above in Sec. IV must balance two potentially competing constraints. First, they must comply with the necessity to keep the achiral potential energy unmodified that, as we discuss above, demands us to decrease the amplitude of the interferences sufficiently so that the optical potential takes a double-well profile at the minimum of $W_{\text {trap }}(\mathbf{r})$. Then, because chiral optical forces
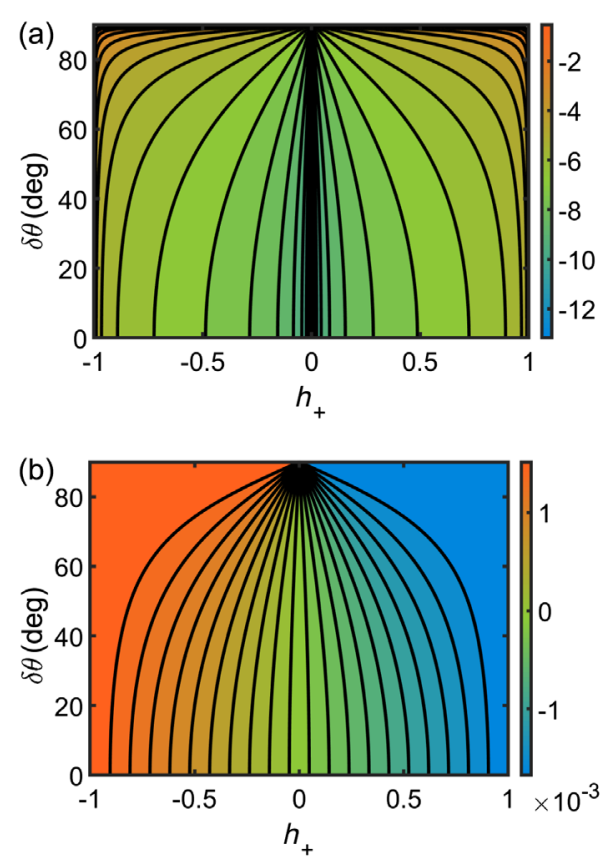

FIG. 5. (a) Surface plot in the $\left(h_{+}, \delta \theta\right)$ plane of $\log \left[\max \left(\mathbf{F}_{\chi}^{\text {reac }}\right) / \max \left(\mathbf{F}_{\text {inter }}\right)\right]$ evaluated on the optical axis in a purely dissipative chiral force configuration. (b) Surface plot in the $\left(h_{+}, \delta \theta\right)$ plane of $\max \left(\mathbf{F}_{\chi}^{\text {diss }}\right) / \max \left(\mathbf{F}_{\text {inter }}\right)$ evaluated on the optical axis in a purely dissipative chiral force configuration. In both reactive and dissipative couplings, $\delta \theta$ and $h_{+}$are chosen in order to maximize the ratio of chiral vs interferential forces while keeping the interferential forces sufficiently weak as to ensure the double-well structure described in Fig. 3. For these calculations, the intensities in the two beams, the wavelength, and the nanosphere dipolar polarizability are the same as in Fig. 3. We set the chiral polarizability $\chi / \alpha=5 \%$ as we discuss in Appendix B.

are weak signals, polarization settings have to allow for an optimal ratio between chiral and interferential axial forces, the latter corresponding to $\mathbf{F}_{\text {inter }}(\mathbf{r})=\operatorname{Re}[\alpha] \nabla W_{\text {inter }}(\mathbf{r})$. The ratio associated with chiral reactive and dissipative forces is plotted in Figs. 5(a) and 5(b), respectively, in the $\left(h_{+}, \delta \theta\right)$ parameter plane, considering that $\delta$ is tuned to shape an achiral potential energy surface symmetrical with respect to the plane $z=0$. For the reactive coupling that involves $K(\mathbf{r})$, the optimal choice would be to set $h_{+}=-h_{-}= \pm 1$ with $\delta \theta=\pi / 2$. However, in this case, no interference is expected, therefore losing the double-well structure. This demands a slight move away from $\delta \theta=$ $\pi / 2$ while reducing the helicity of the two beams. In contrast, the dissipative coupling involves $\boldsymbol{\Phi}(\mathbf{r})$ where $h_{+}=h_{-}$maximizes interferential forces associated with very deep wells in the potential energy. Our choice here is rather to set $\delta \theta=\pi / 2$ with a reduced helicity in both beams. These constraints lead to the polarization choices detailed in the caption of Fig. 3 that yield force ratios strong enough for our purposes while preserving the double-well profile of $W_{E}(\mathbf{r})$. 


\section{THERMODYNAMIC CONSEQUENCES OF THE REACTIVE AND DISSIPATIVE CHIRAL COUPLINGS}

We now include temperature $T$ and describe the evolution of the dipole inside the bistable potential. In the absence of chiral contributions $[K(\mathbf{r})=0, \boldsymbol{\Phi}(\mathbf{r})=\mathbf{0}]$, the evolution is driven only by decoupled achiral reactive axial $\mathbf{F}_{\alpha}(\mathbf{r}) \cdot \hat{\mathbf{z}}$ and radial $\mathbf{F}_{\alpha}(\mathbf{r}) \cdot \hat{\boldsymbol{\rho}}$ forces inside the optical trap. This situation corresponds to a Kramers problem with the possibility given to the dipole to escape local trapping sites by thermal activation and diffusion over the separating barrier of the double-well potential energy landscape drawn in Fig. 3. The classical overdamped Fokker-Planck model for this metastable dynamics is reviewed in Appendix D, with the escape rates $\kappa_{A \rightarrow C}$ from well $A$ to well $C$ and $\kappa_{C \rightarrow A}$ from well $C$ to well $A$ given by the well-known formulas

$$
\begin{aligned}
& \kappa_{A \rightarrow C} \simeq \frac{a b}{k_{B} T \gamma \pi} e^{-\left[U_{\mathrm{opt}}\left(0, z_{B}\right)-U_{\mathrm{opt}}\left(0, z_{A}\right) / k_{B} T\right]}, \\
& \kappa_{C \rightarrow A} \simeq \frac{b c}{k_{B} T \gamma \pi} e^{-\left[U_{\mathrm{opt}}\left(0, z_{B}\right)-U_{\mathrm{opt}}\left(0, z_{C}\right) / k_{B} T\right]}
\end{aligned}
$$

where $k_{B}$ is the Boltzmann constant and $\gamma$ the Stokes drag coefficient. These formulas involve the local $a, c$ minima and barrier $b$ curvatures together with the barrier heights $\Delta U_{\mathrm{opt}}^{A B}=U_{\mathrm{opt}}\left(0, z_{B}\right)-U_{\mathrm{opt}}\left(0, z_{A}\right)$ and $\Delta U_{\mathrm{opt}}^{C B}=U_{\mathrm{opt}}\left(0, z_{C}\right)-$ $U_{\text {opt }}\left(0, z_{B}\right)$, both evaluated along the optical axis at $q=0$.

In the following subsections, we evaluate the thermodynamic consequences of the chiral coupling by looking at how the escape rates are modified due to the presence of the reactive and dissipative chiral optical forces in the FokkerPlanck equation. In our model, we look only at the force contributions along the optical axis. This is natural due to the axisymmetry of the system. The relevant dynamics is thus described through axial force components only and close to the optical axis. It is, however, important to keep in mind that the conservative and nonconservative nature of the forces is a known input of the problem from Eqs. (15) and (16). This is important when modeling the case of dissipative coupling below. We show that fully three-dimensional stochastic simulations presented in Sec. VII validate our model that focuses on the predominant axial thermodynamics.

\section{A. Steady state in the reactive chiral coupling}

Here, we select the polarizations $h_{+}=h_{-}$in the two beams in order to induce a purely reactive chiral coupling and to study its impact on the thermodynamics of the thermal activation process inside the bistable optical trap. In this case, the reactive chiral optical force derives from the gradient of the chirality density and is thus conservative. It therefore contributes to the optical energy potential as a chiral potential

$$
U_{\chi}(q, z)=-\operatorname{Re}[\chi] K(q, z) / \omega \sqrt{\varepsilon_{f} \mu_{f}} .
$$

We stress that this contribution built on the pseudo-scalar $K(q, z)$ corresponding to the chiral density of the electromagnetic field is a potential intrinsically truly chiral. This is a surprising feature that must be well appreciated. The potential is not chiral by virtue of the chirality of the dipole: Its enantiomorphism stems from the chirality of the electromagnetic field only.

This chiral potential $U_{\chi}(q, z)$ then adds to the dynamics described by the steady-state Fokker-Planck equation according to (forward direction)

$$
\begin{aligned}
\tilde{j}_{z}^{+}(q)= & -\frac{1}{\gamma} \partial_{z} U_{\mathrm{pot}}(q, z) \tilde{p}^{+}(q, z) \\
& -D \partial_{z} \tilde{p}^{+}(q, z)
\end{aligned}
$$

defining $U_{\text {pot }}(q, z)=U_{\text {opt }}(q, z)+U_{\chi}(q, z)$. The equation connects the probability density $\tilde{p}^{+}$to the probability current $\tilde{j}_{z}^{+}$both modified by the chiral potential from the probability density $p^{+}$and the probability current $j_{z}^{+}$of the bare Kramers problem (see Appendix D). In this model, we involve only the probability current along the optical axis $\tilde{j}_{z}^{+}(q)$. This projective approach gives us an efficient way to analytically interpret the thermodynamic impact of the chiral potential through well-identified contributions. We describe below a full modeling of the system resorting to numerical solutions of 3D Langevin equations under chiral coupling. Anticipating with what we show below, these 3D numerical simulations fully justify our projective approach, thereby confirming that the $z$-axial component of the probability current dominates the global dynamics and therefore the thermodynamics.

Just like $\tilde{j}_{z}^{+}(q)$ is modified by $U_{\chi}(q, z)$, the nonequilibrium probability density is modified with respect to the bare Kramers problem into $\tilde{p}^{+}$and yields escape rates that evolve accordingly with

$$
\begin{aligned}
& \tilde{\kappa}_{A \rightarrow C} \simeq \kappa_{A \rightarrow C} \times e^{-\left[U_{\chi}\left(0, z_{B}\right)-U_{\chi}\left(0, z_{A}\right) / k_{B} T\right]}, \\
& \tilde{\kappa}_{C \rightarrow A} \simeq \kappa_{C \rightarrow A} \times e^{-\left[U_{\chi}\left(0, z_{B}\right)-U_{\chi}\left(0, z_{C}\right) / k_{B} T\right]},
\end{aligned}
$$

where we verify that the local curvatures of the optical landscape are only weakly modified by the chiral potential with respect to the results of Appendix D, i.e., that $\tilde{a} \simeq a$, $\tilde{b} \simeq b$, and $\tilde{c} \simeq c$. We use the same notation for $U_{\chi}$ as we use for $U_{\text {opt }}$ above, and where we take advantage of the $z$ parity of the chiral density $K(q, z)$ with $\left.\partial_{z} U_{\chi}(z)\right|_{z=0}=0$ and its $q$ parity giving $\left(\partial^{2} U_{\text {opt }} / \partial q \partial z\right)(q, z) \sim 0$ close to the optical axis. This parity also implies that the reactive chiral coupling does not lift the degeneracy in free energy between the two wells, maintaining the equilibrium constant to $\tilde{\kappa}_{A \rightarrow C} / \tilde{\kappa}_{C \rightarrow A}=1$.

From a thermodynamics viewpoint, the rate modifications come from the work performed by the reactive chiral force between the barrier and the wells. This conservative work provides a contribution to the potential energy in the 

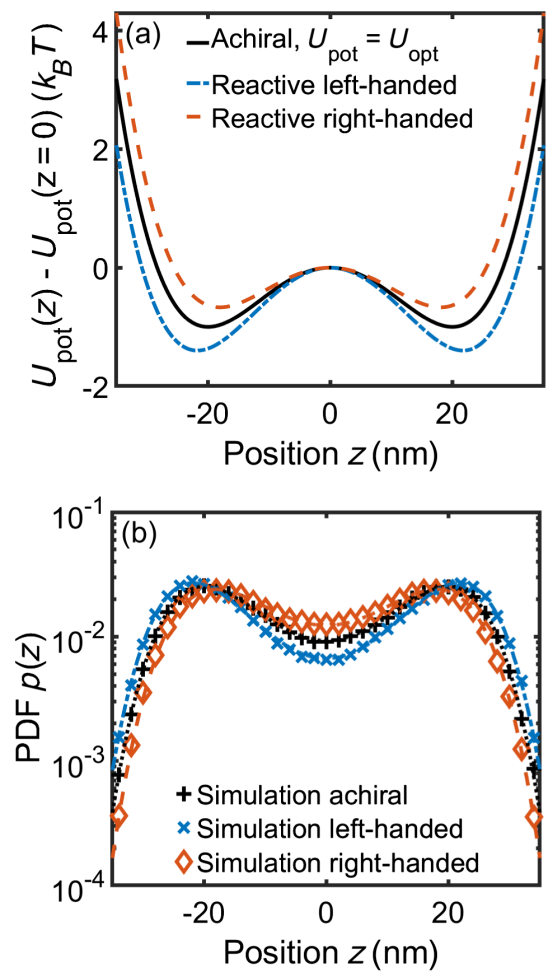

FIG. 6. (a) With $h_{+}=h_{-}$and the polarization settings of the two beams detailed in Fig. 3, the chiral coupling is reactive, leading to conservative chiral optical forces exerted on the chiral dipolar nanosphere $(\chi / \alpha=5 \%)$ diffusing within the bistable optical trap. Because these forces derive from a chiral potential $U_{\chi}(q, z)$, they combine with the achiral electromagnetic potential to form the potential energy surface $U_{\text {pot }}(q, z)$. The resulting potentials at $q=0$ are plotted for the two opposite enantiomers: the right-handed $(\operatorname{Re}[\chi]>0$ in red) and the lefthanded one $(\operatorname{Re}[\chi]<0$ in blue). The same is plotted in black for an achiral environment. The same differences would be induced for one fixed choice of enantiomer but using two opposite enantiomorphs for the reactive chiral optical field. (b) The corresponding normalized PDFs are evaluated from our onedimensional model (lines) and three-dimensional simulations (symbols) as detailed in Appendix E. Although the achiral and chiral PDFs appear only slightly different, their enantioselective character is manifest, revealing a chiral discriminating thermodynamics.

form of a Helmholtz free-energy difference $\Delta \mathcal{F}_{\chi i}=W_{\chi i}^{\text {cons }}=$ $U_{\chi}\left(z_{B}\right)-U_{\chi}\left(z_{i}\right)$, with $i=A, C$.

The second important thermodynamic consequence is the enantioselective character of the free-energy difference $\Delta \mathcal{F}_{\chi}$ considering that $\operatorname{Re}[\chi]$ has opposite signs for different enantiomers of the chiral dipole and that $K(q, z)$ being a pseudo-scalar changes sign for the enantiomorphs (parity operation) of the chiral optical standing wave. Chiral coupling therefore has the capacity to yield a new potential energy surface that depends on both the chirality of the dipole and of the optical field. This dual enantiomeric and enantiomorphic dependence of $W_{\chi}^{\text {cons }}$ is the manifestation of a truly chiral discriminating thermodynamic process, concentrating one enantiomer toward the center and the other toward the outside of the double well as illustrated in Fig. 6.

Figure 6(a) indeed displays the initial optical potential energy and the changes induced on it by the chiral density $K(\mathbf{r})$ through the chiral coupling. As seen in Fig. 6(a), the contribution of the chiral potential proportional to $W_{\text {trap }}(\mathbf{r})$ with a sign determined by the enantiomeric form of the dipole, either enhances the trapping component of $W_{E}(\mathbf{r})$ for right-handed eniantomers $(\operatorname{Re}[\chi]>0)$ or favors its interferential component $W_{\text {inter }}(\mathbf{r})$ for left-handed ones $(\operatorname{Re}[\chi]<0)$.

In the steady-state regime that implies the detailed balance, we also plot in Fig. 6(b) the probability density function (PDF) evaluated on the optical axis at $q=0$, which is simply given by

$$
p_{\chi}^{\text {reac }}(0, z)=C e^{-\left[U_{\text {opt }}(0, z)+U_{\chi}(0, z) / k_{B} T\right]},
$$

with $U_{\text {opt }}(0, z)=-\operatorname{Re}[\alpha] W_{E}(0, z), U_{\chi}(0, z)=-\operatorname{Re}[\chi] K(0, z) /$ $\omega \sqrt{\varepsilon_{f} \mu_{f}}$, and $C$ a normalization factor evaluated such that $\int_{-\infty}^{+\infty} d z p_{\chi}^{\text {reac }}(0, z)=1$.

\section{B. Steady state in the dissipative chiral coupling}

If we change the polarizations to $h_{+}=-h_{-}$, the standing wave now carries a chiral flux with no chiral density. As a consequence, a dissipative chiral force is exerted on the diffusing chiral dipole. As we explain below, this mere change of polarization that switches the chiral optical environment from reactive to dissipative leads to a totally different thermodynamics.

Because the dissipative chiral force is nonconservative with $\boldsymbol{\nabla} \times \mathbf{F}_{\chi}^{\text {diss }}(q, z) \neq \mathbf{0}$, it is not possible to derive it from a chiral potential, as it is the case for the reactive chiral coupling. But despite the nonconservative nature of the chiral force, we solve the steady-state Fokker-Planck equation with the modified probability density $\hat{p}^{+}(q, z)$ and the probability current $\hat{j}_{z}^{+}(q)$ along the optical axis

$$
\begin{aligned}
\hat{j}_{z}^{+}(q)= & -\frac{1}{\gamma}\left[\partial_{z} U_{\mathrm{opt}}(q, z)-\mathbf{F}_{\chi}^{\mathrm{diss}}(q, z) \cdot \mathbf{z}\right] \hat{p}^{+}(q, z) \\
& -D \partial_{z} \hat{p}^{+}(q, z) .
\end{aligned}
$$

The equation is solved analytically by making use of the fact that under the paraxial approximation, the $z$ dependence of the chiral flux $\boldsymbol{\Phi}(q, z)$ is very slow over the distance $\Delta z \sim \Delta \ell$ separating the two local minima. The projected chiral dissipative force $F_{\chi}^{\text {diss }}(q, z)=\hat{\mathbf{z}} \cdot \mathbf{F}_{\chi}^{\text {diss }}(q, z)$ is thus such that

$$
\Delta F_{\chi}^{\text {diss }}(q, \Delta z)=\partial_{z}^{2} F_{\chi}^{\text {diss }}(q, 0) \Delta z^{2} \ll F_{\chi}^{\text {diss }}(q, 0)
$$

given that the symmetry of the force field imposes $\partial_{z} F_{\chi}^{\text {diss }}(q, 0)=0$. This is well seen in Fig. 7(a), where 

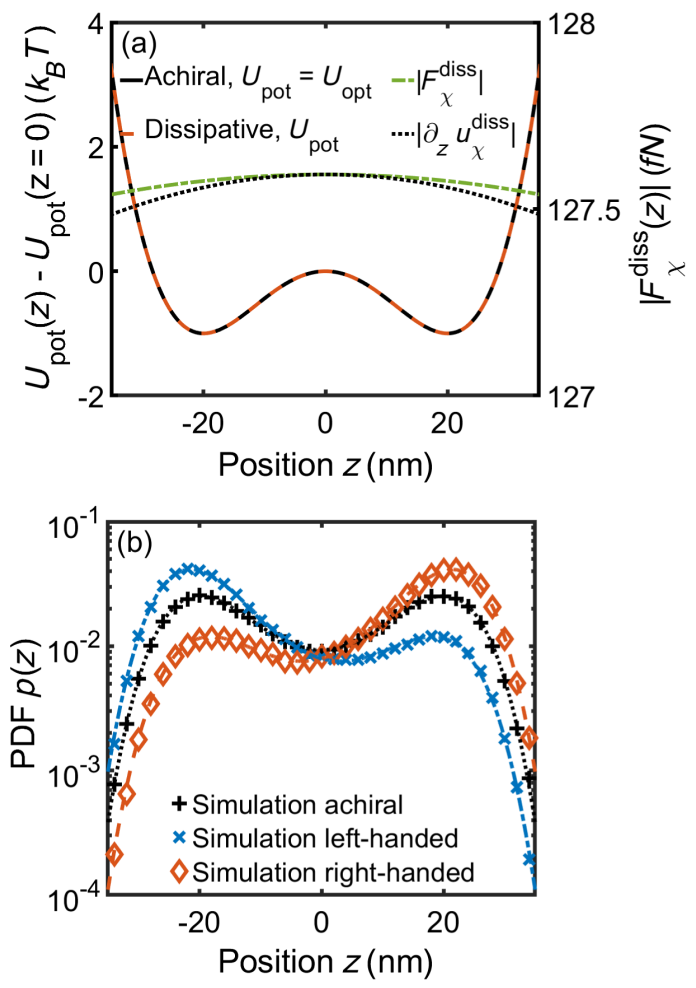

FIG. 7. (a) A dissipative chiral coupling is induced by the chiral nanosphere $(\chi / \alpha=5 \%)$ for $h_{+}=-h_{-}$in the two beams and the polarization settings detailed in Fig. 3 . The angle formed between the polarization axes of the beams and their helicities are fixed so as to lead to the generation of a chiral flux $\boldsymbol{\Phi}(q, z)$ - and zero chiral density - with the same achiral optical potential energy density $U_{\text {opt }}(q, z)$ as for an achiral environment [in black, same as in Fig. 3(a)] again calculated at $q=0$. Our chiral dipole now couples to the chiral optical environment through $\operatorname{Im}[\chi]$ with chiral dissipative forces $F_{\chi}^{\text {diss }}(q, z)$ that are opposed for opposite signs in $\operatorname{Im}[\chi]$ - the same sign inversion appears if, instead of changing dipole enantiomers, one changes electromagnetic field enantiomorphs. Note that in the paraxial approximation of the model with $\Delta \ell \ll z_{R}$, we have $\Delta F_{\chi}^{\text {diss }}(q, z) \ll F_{\chi}^{\text {diss }}(q, z)$; see the main text. Here, we plot the profile of a second-order development of $F_{\chi}^{\text {diss }}(q, z)$ (black line) with the associated scale on the right-hand side of the graph. Because the dissipative chiral force is nonconservative, it does not contribute to the potential freeenergy surface as it is the case for the reactive chiral coupling. (b) The influence of the chiral dissipative force is seen on the modified steady-state probability density function of the chiral dipole in the bistable trap, shifted in the direction of the force for both our one-dimensional model (lines) and three-dimensional simulations (symbols); see details in Appendix E. This modified PDF reveals the strong chiral discriminating action of $F_{\chi}^{\text {diss }}(0, z)$ with respect to the two local maxima at $z_{A}$ and $z_{C}$. We give in the main text the thermodynamics interpretation of this result.

over $\Delta z \approx 100 \mathrm{~nm},\left|F_{\chi}^{\text {diss }}(0, \Delta z)-F_{\chi}^{\text {diss }}(0,0)\right| /\left|F_{\chi}^{\text {diss }}(0,0)\right|=$ $\Delta z^{2} / z_{R}^{2} \approx 3.4 \times 10^{-3}$.

This slow-varying evolution of $F_{\chi}^{\text {diss }}(q, z)$ throughout the bistable region makes it possible to approximate the dissipative force by its Taylor expansion around $(q, z=0)$ in $z$. Given the parity of the force, only pair orders are present, and coefficients evolve in $1 /\left(z_{R}^{2 n}\right)$ where $n$ is the expansion order in $z$. Choosing an arbitrary expansion order, we can tune the precision of the approximation of the chiral force field over a given volume inside the trap. This expansion can then be integrated as a pseudopotential $u_{\chi}^{\text {diss }}(q, z)$, while keeping in mind that this is only locally possible. As stressed above, no chiral potential can be derived from the dissipative chiral force, which is strictly nonconservative with $\boldsymbol{\nabla} \times \mathbf{F}_{\chi}^{\text {diss }}(q, z) \neq \mathbf{0}$.

Our approximation therefore neglects the fact that the pseudo-potential defined is dependent on $q$ while there is no associated radial force. In an effective way, we use a pseudoone-dimensional model with a radial $q$ parameter, exploiting the fact that in one dimension, all forces can be derived from a potential. For the sake of simplicity, we use a second-order development for our model for defining $F_{\chi}^{\text {diss }}(q, z)=$ $-\partial_{z} u_{\chi}^{\text {diss }}(q, z)$. This pseudo-potential approach helps us solve the steady-state Fokker-Planck equation (22) using the same steepest-descent approach and the $q$ parity of $\boldsymbol{\Phi}(q, z)$. We can then evaluate analytically the probability density function under dissipative chiral coupling plotted in Fig. 7(b); see below.

Under such an approximation, the Fokker-Planck equation (22) is directly integrated, leading to escape rates modified by the external chiral dissipative force field as

$$
\begin{aligned}
& \hat{\kappa}_{A \rightarrow C} \simeq \kappa_{A \rightarrow C} \times e^{+\left[F_{\chi}^{\text {diss }}(0,0)\left(z_{B}-z_{A}\right) / k_{B} T\right]}, \\
& \hat{\kappa}_{C \rightarrow A} \simeq \kappa_{C \rightarrow A} \times e^{+\left[F_{X}^{\text {diss }}(0,0)\left(z_{B}-z_{C}\right) / k_{B} T\right]},
\end{aligned}
$$

with $\hat{a} \simeq a, \hat{b} \simeq b$, and $\hat{c} \simeq c$ as we verify here too. The fact that the $q$ dependence has disappeared from the rate expressions is the result of our first-order approximation. As a consequence, these expressions look as if they could have been derived within a chiral conservative force field. But it is crucial to appreciate that we already know from Eq. (16), that the chiral force here is nonconservative. This prior knowledge is fundamental in that it completely modifies the thermodynamics that must be associated with Eqs. (24) and (25) with respect to the conservative case.

Here indeed, the chiral electromagnetic fields continuously transfer, through dissipation, mechanical energy to the chiral dipole immersed in this dissipative chiral environment. For this reason, our system behaves as a nonequilibrium steady-state system where the chirality of the probe becomes a thermodynamic parameter. The thermodynamic consequence of the emergence of a dissipative chiral optical force is a bias put on the probability distribution function of positions from both sides of the waist. In this dissipative coupling, the PDF is evaluated in the stationary regime on the optical axis using a 
nonequilibrium potential $p_{\chi}^{\text {diss }}(0, z)=C \exp [-\varphi(0, z)]$ where we have, within the pseudo-potential $u_{\chi}^{\text {diss }}(0, z)$ approach, $\varphi(0, z)=\left[U_{\mathrm{opt}}(0, z)+u_{\chi}^{\mathrm{diss}}(0, z)\right] / k_{B} T$ and the normalization $C^{-1}=\int_{-\infty}^{+\infty} d z \exp [-\varphi(0, z)][37,38]$. It is plotted in Fig. 7(b).

As already emphasized, the chiral coupling intertwines the chirality of the dipole with the chirality of the field, while leaving untouched the achiral bistable potential. For this reason, the bias depends on both the enantiomeric form of the dipole via $\operatorname{Im}[\chi]$ and the enantiomorphic form of the field through the chiral flux $\boldsymbol{\Phi}(q, z)$. But contrasting the reactive case, the dissipative chiral action cannot be framed into a chiral contribution to the potential energy landscape. In such a framework, the nonconservative chiral force contributes to the thermodynamics as a dissipative work $F_{\chi}^{\text {diss }}(q, z) \delta z=\delta W_{\chi}^{\text {diss }}$ and not as a free-energy change [61]. This fundamental difference in the thermodynamics between the reactive and the dissipative chiral couplings has important consequences as we now see.

The fact that the dissipative chiral force breaks the symmetry of the escape rates according to

$$
\frac{\hat{\kappa}_{A \rightarrow C}}{\hat{\kappa}_{C \rightarrow A}}=e^{+\left[F_{X}^{\text {diss }}(0,0)\left(z_{C}-z_{A}\right) / k_{B} T\right]}
$$

constitutes the main result of our analysis. It explicitly shows how dissipative chiral coupling lifts the degeneracy in the escape rates of the initial system. This result has a profound thermodynamical significance by showing how dissipative chiral coupling acts as a chiral source of heat, with a quantity

$$
\Delta Q_{\chi}=F_{\chi}^{\mathrm{diss}}(0,0) \Delta \ell
$$

transferred to the surrounding fluid in the trap that can be precisely evaluated from well to well with $\Delta \ell=z_{C}-z_{A}$. Equations (24) and (25) show the dissipative work associated with each barrier crossing $\pm F_{\chi}^{\text {diss }}(0,0) \Delta \ell / 2$ (we note here $\Delta \ell / 2=z_{B}-z_{A}>0$ ). Then, the symmetry breaking of the escape rates shown in Eq. (26) stems from the total dissipative work evaluated from one well to the other. This global dissipative work is associated with a quantity that corresponds to the quantity of heat (27) transferred to the bath. The consequence is observed in Fig. 7(b) in the difference in the probability density function between the two wells. The asymmetry of the PDF ensures that this heat $\Delta Q_{\chi}$ is, on average, not fully recovered by the particle.

It is important to stress that in our dipolar framework, this heat cannot come from a transfer from a "laser-heated" particle to the fluid, but only from the damping through friction of the stochastic motion of the chiral dipole performed under the continuous action of the external dissipative chiral force field. It is also important to note that in our model, the heat associated with $q \neq 0$ contributions is not accounted for. These off-axis contributions, however, play a negligible role in the modifications of probability density functions and escape rates. This is seen by looking the excellent agreement between our model in Fig. 7(b) and full 3D simulations that obviously incorporate all $q \neq 0$ contributions. This agreement confirms that it is possible to evaluate the transfer of heat along the optical axis, as done in our model Eq. (27).

The heat transfer can be described as an associated entropy production $\Delta S_{\chi}=\Delta Q_{\chi} / T$ during the diffusion of the dipole from one well to the other. This production of entropy is only related to the dissipative chiral dynamics that assigns enantiomers to specific wells of the bistable potential. Through Brownian diffusion performed within this dissipative optical landscape, the chiral coupling leads to spatially separating the enantiomers. This is exactly a deracemization process, and this production of entropy can be associated with the thermodynamic cost of assigning one enantiomer to one specific well of the bistable potential. As such, it is the exact analog to the "entropy penalty" expected for any molecular deracemization process, as we mention in Sec. II.

Finally, we want to stress that the agreement seen in Fig. 7(b) for the PDF profiles between the 3D stochastic simulations and our model confirms the validity of locally deriving the dissipative chiral force field from a pseudopotential. It demonstrates also that our model captures correctly the heat (and thus, the produced entropy) transferred during the diffusion of the dipole from one well to the other along the optical axis. But one should keep in mind that our pseudo-potential approach is locally accessible looking only at the on-axis probability current. In contrast, our full 3D simulations do not rely on this approach and are handling rigorously the nonconservative property of the dissipative chiral force field. This therefore implies that in the 3D description of our system below, the influence of the dissipative chiral force field cannot be described as deriving from a tilted potential that breaks the symmetry between the two wells. We also note that the nonconservative chiral force field has no azimuthal components. Azimuthal components solely stem from the achiral contribution and therefore do not influence the impact of the chiral coupling on the thermodynamics of the barrier crossing.

\section{STOCHASTIC SIMULATIONS: TRAJECTORIES AND PROBABILITY DENSITY FUNCTIONS}

Once the model is exposed and analytically solved, it is important to validate the results shown in Figs. 6 and 7 by simulating the three-dimensional instantaneous motion $\mathbf{r}(t)$ of the chiral dipole inside the optical trap when the polarizations are set to induce chiral optical environments. Such 3D simulations will also lead us a step further by revealing the different patterns that chiral separation 
mechanisms can spatially yield within reactive or dissipative environments.

To do so, we solve the overdamped Langevin equation

$$
\gamma d_{t} \mathbf{r}=-\nabla U_{\mathrm{opt}}(\mathbf{r})+\mathbf{F}_{\chi}(\mathbf{r})+\mathbf{F}_{\mathrm{th}}(t)
$$

in the achiral bistable optical potential $U_{\text {opt }}(\mathbf{r})$ with $\mathbf{F}_{\mathrm{th}}(t)$ the thermal random force of zero mean that satisfies the fluctuation-dissipation theorem. We include in this Langevin equation the three-dimensional chiral force fields $\mathbf{F}_{\chi}(\mathbf{r})$ (axial and radial) whose expressions are reminded in Sec. III. In Fig. 3(a), the electromagnetic field intensity is adjusted so that the bistable barrier separating the two local potential minima is set to a height of one $k_{B} T$.

The simulations are performed in achiral $\left[\mathbf{F}_{\gamma}(\mathbf{r})=\mathbf{0}\right]$, chiral reactive $\left[\mathbf{F}_{\chi}(\mathbf{r})=\mathbf{F}_{\chi}^{\text {reac }}(\mathbf{r})\right]$, and chiral dissipative $\left[\mathbf{F}_{\chi}(\mathbf{r})=\mathbf{F}_{\chi}^{\text {diss }}(\mathbf{r})\right]$ configurations using the same polarization settings as those involved in Fig. 3. Again, the chirality of the trapped nanosphere is set to $\chi / \alpha=5 \%$. Simulations are run for a racemic mixture of chiral dipoles corresponding to $10^{4}$ trajectories per enantiomer in parallel, starting from an initial distribution of positions determined from the threedimensional stationary probability density distributions evaluated by our model for $5 \times 10^{4}$ time steps. Simulation algorithms and methods are detailed in Appendix E.

Within all the available states that lie below the level set by the temperature and the simulation time, these results perfectly reveal how the Brownian motion probes the chiral optical environment, where the chiral coupling modifies the diffusion driven by thermal fluctuations within the bistable potential. The spatial distributions of positions numerically calculated and shown in Fig. 8 clearly reveal this bias. In the achiral case of Fig. 8(a), the distribution does not depend on the enantiomer while both reactive [Fig. 8(b)] and dissipative [Fig. 8(c)] cases are enantiodependent. With the chosen optical enantiomorph, we see in the reactive case that an optically trapped right-handed enantiomer with $\operatorname{Re}[\chi]>0$ is more concentrated toward the trapping maximum than it is for the opposite $\operatorname{Re}[\chi]<0$ enantiomer. In the dissipative coupling, the enantiomers are clearly spatially separated. These signatures seen on trajectories complement Sec. VI B in the demonstration and characterization of a genuine optomechanical deracemization process.

For each of the three simulations, we build the corresponding PDF and compare them in Figs. 6(b) and 7(b) with the simulated PDFs to the model axial PDFs, respectively, $p_{\chi}^{\text {reac }}(0, z)$ and $p_{\chi}^{\text {diss }}(0, z)$. The excellent agreement validates in the specific case of dissipative chiral coupling, the pseudo-potential approach of our model. It also validates for all cases that one can use the corresponding stationary PDF of the model for initializing the simulations, as we discuss in Appendix E.
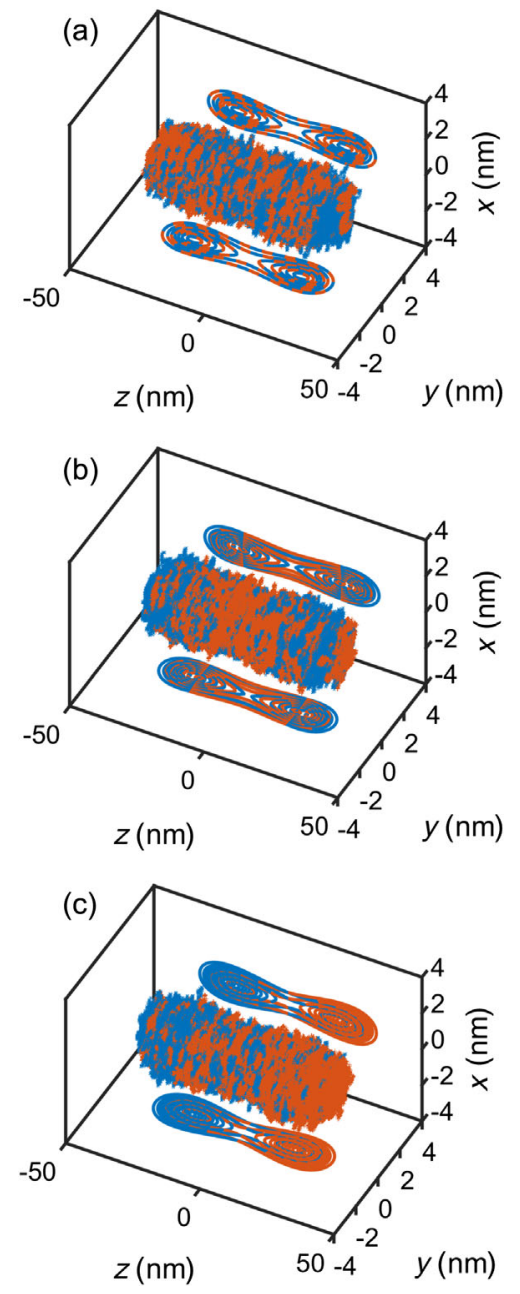

FIG. 8. Time-dependent positions of $R=20 \mathrm{~nm} \mathrm{Au} \mathrm{nano-}$ spheres in a racemic mixture of 100 left-handed (in blue) and right-handed (in red) enantiomers simulated for 50000 time steps of $95.4 \mathrm{ps}$ for a total of $4.77 \mu \mathrm{s}$. These trajectories are randomly picked among the $10^{4}$ trajectories used to form the simulated PDF displayed in Figs. 6(b) and 7(b). We vary the chiral nature of the optical environment going through (a) an achiral optical environment, (b) a reactive chiral optical environment, and (c) a dissipative chiral optical environment. We can see that in the achiral case, the distribution is totally independent from the enantiomeric form of the nanoparticle, as expected. In the reactive case, in contrast, the chiral nanoparticles are either more concentrated toward the center of the optical trap for the family of right-handed enantiomers $(\operatorname{Re}[\chi]>0)$ or moved away to external regions for the left-handed enantiomers $(\operatorname{Re}[\chi]<0)$. In the dissipative case, the chiral coupling is capable of inducing a deracemization process by progressively localizing enantiomers to different wells in strict relation with their enantiomeric $\operatorname{Im}[\chi] \lessgtr 0$ forms. For each of the different types of couplings, the contour plots shown on the axis planes correspond to the predictions of the $p_{\text {opt }}(q, z), p_{\chi}^{\text {reac }}(q, z), p_{\chi}^{\text {diss }}(q, z)$ PDFs given by our one-dimensional model. As well seen, our model successfully reproduces both the axial and radial distributions. 


\section{STOCHASTIC SIMULATIONS: WELL RESIDENCY TIMES STATISTICS}

We now look at single long diffusive trajectories of one chiral nanosphere within the optical trap, thermally activated from one local well to the other. The unique capacity, presented just above, of our 3D simulations for visualization is complemented here by a dynamic analysis performed on the escape rates. Simulating the fluctuation spectra of the escape rates will provide a striking characterization of the chiral deracemization process from the stochastic point of view.

For such a study, it is important to have good statistics on barrier-crossing events, and we therefore choose to use here a number of trajectories reduced in comparison with the ensemble simulations of Sec. VII but allowing us to calculate over longer times-over 2.9 ms corresponding to $3 \times 10^{6}$ points with a time step $d t=0.95 \mathrm{~ns}$.

Figure 9(a) shows a time trace $z(t)$ of one such long diffusing trajectory in the achiral bistable potential defined in Sec. IV. The time trace clearly reveals the stochastic motion of the trapped nanoparticle that "jumps" from one well to the other (ca. 40 jumps for a 2.9 -ms trajectory). Such jumps are described by a Poisson statistics where the residency time $\tau_{i}$ in each well $i=A, C$ follows an exponential law $P\left(\tau_{i}\right)=\exp \left(-\tau_{i} /\left\langle\tau_{i}\right\rangle\right) /\left\langle\tau_{i}\right\rangle$, where $\left\langle\tau_{i}\right\rangle$ is the mean residency time in well $A$ or $C$ [62]. As we explain in Appendix F, the evaluation of such distributions demands a careful identification of the jumps, accounting for the possible recrossing events present in all thermally
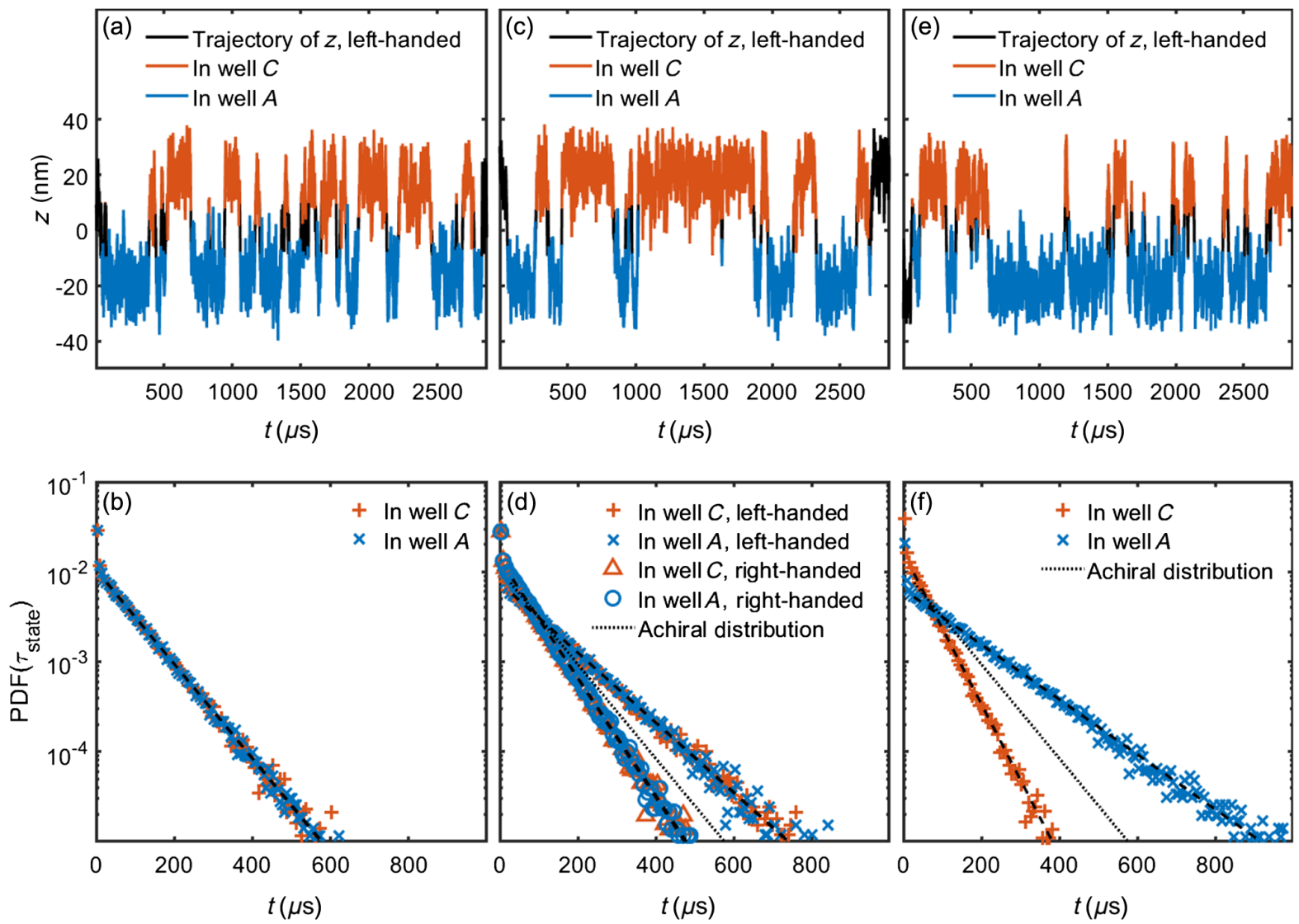

FIG. 9. (a) One trajectory simulated over $2.9 \mathrm{~ms}$ with time steps of $0.95 \mathrm{~ns}$ corresponding to the diffusion of an optically trapped nanosphere in the achiral bistable optical potential energy landscape displayed in Fig. 3(b). Jumps are identified using a hysteresis of $\sigma=10 \mathrm{~nm}$. The trapped periods are highlighted for well $A$ in blue and $C$ in red. The first and last events are excluded as we explain in Appendix F. (b) Cumulated statistics (over 4096 trajectories) of the residency time in both wells $A(z<0$, in blue) and $C$ ( $z>0$, in red) for the symmetric bistable potential. For times larger than the relaxation time of the well, the statistics follow a Poisson distribution, as expected from Kramers theory. The average residency time in one well corresponds to the inverse of the linear slope of the distribution associated with that well, plotted in a linear-log scale. (c) One trajectory simulated within a chiral reactive environment for a left-handed chiral nanosphere $(\operatorname{Re}[\chi]<0)$. As can be seen, the nanosphere spends an equivalent time in both wells, but its jumps frequency is slowed down compared to the achiral case. As seen in (d), the same phenomenon appears with the same degeneracy but with an acceleration for a right-handed nanosphere. (e) One trajectory, this time simulated within a chiral dissipative environment for a left-handed chiral nanosphere $(\operatorname{Im}[\chi]>0)$. As can be seen, the chiral nanosphere does not spend an even time between the two wells, in stark contrast with both the achiral bistable case and the chiral reactive coupling case. The breaking of symmetry when the dissipative chiral coupling is switched on is reflected in the splitted exponential laws associated with the two wells calculated in (f). 
activated barrier-crossing diffusive systems. Figure 9(b) shows that the symmetry of the achiral bistable potential leads to identical exponential laws for the residency times inside each well $A$ and $C$.

A time trace $z(t)$ simulated in the case of a reactive chiral coupling is displayed in Fig. 9(c). The degeneracy in residency times preserved in the reactive chiral coupling is clearly observed in Fig. 9(d), with a difference in the residency times corresponding to the differences in the well depths shown in Fig. 6. The discriminative action of the chiral reactive coupling is measured here on the exponent differences between the two enantiomer families.

In contrast, in the case of dissipative chiral coupling, we already know from Sec. VI B that the degeneracy between the two wells is broken. This is perfectly seen on the time trace of the diffusion dynamics of the chiral nanosphere displayed in Fig. 9(e) and more clearly on the probability distributions of the residency times in Fig. 9(f). The observed tendency to spend more time in well $A$ than in well $C$ for a left-handed enantiomer $(\operatorname{Im}[\chi]>0)$ is in agreement with the probability density functions plotted in Fig. 7(b). This symmetry breaking is responsible for the deracemization process observed in Fig. 8(c) above when dealing with a pure racemic mixture composed of a large even number of optically trapped left- and right-handed enantiomers. In this dissipative case, we measure from the exponential laws a ratio between the residency times $\left\langle\tau_{C}^{\text {diss }}\right\rangle /\left\langle\tau_{A}^{\text {diss }}\right\rangle=0.36$.

This measured ratio can be directly compared to the model with $\left\langle\tau_{C}^{\text {diss }}\right\rangle /\left\langle\tau_{A}^{\text {diss }}\right\rangle=\hat{\kappa}_{A \rightarrow C} / \hat{\kappa}_{C \rightarrow A}$. The ratio is evaluated using Eq. (26), reaching $\hat{\kappa}_{A \rightarrow C} /\left.\hat{\kappa}_{C \rightarrow A}\right|_{\text {model }}=0.29$, which clearly departs from the simulated result. This disagreement suggests that the role of the force-field contributions cannot simply be limited to quadratic approximations taken at the wells' minima, as it is done in the steepest-descent approach of our one-dimensional model. Accounting for higher-order terms, i.e., the anharmonicity of the wells around the barrier, is required for quantitative comparisons.

This is confirmed if we now look at three-dimensional PDFs. This ratio indeed can also be related to the populations inside each well according to $\left\langle\tau_{C}^{\text {diss }}\right\rangle /\left\langle\tau_{A}^{\text {diss }}\right\rangle=\hat{n}_{C}^{-} \hat{J}_{z}^{+} / \hat{n}_{A}^{+} \hat{J}_{z}^{-}$ and therefore can be directly evaluated within the detailed balance $\hat{J}_{z}^{+}=\hat{J}_{z}^{-}$using probability currents. In this approach, we extend the expression of the detailed balance stationary PDF given in Sec. VIB to three dimensions with $p_{\chi}^{\text {diss }}(q, z)=C \exp [-\varphi(q, z)]$, with $\varphi(q, z)=\left[U_{\text {opt }}(q, z)+\right.$ $\left.u_{\chi}^{\text {diss }}(q, z)\right] / k_{B} T$. The population in one well is then evaluated by an integration of the PDF restricted over the well, and therefore,

$$
\frac{\hat{n}_{C}^{-}}{\hat{n}_{A}^{+}}=\frac{\int_{0}^{\infty} d q 2 \pi q \int_{0}^{\infty} d z p_{\chi}^{\mathrm{diss}}(q, z)}{\int_{0}^{\infty} d q 2 \pi q \int_{-\infty}^{0} d z p_{\chi}^{\operatorname{diss}}(q, z)}=0.36 .
$$

The perfect agreement with the simulations confirms the (expected) quantitative importance of accounting for the anharmonic curvature of the potential generated by the interfering Gaussian beams.

The important scope of these results is to show that it is possible to detect and measure the presence of chiral optical forces by looking at the average of the residency times of each of the wells rather than at the forces themselves. Considering that these residency times are exponentially sensitive to either the chiral free energy (in the case of reactive chiral coupling) or the chiral heat (in the case of dissipative chiral coupling), one expects such an approach to yield a high resolution in detection of chiral optical forces and in the resolution of the chiral discriminative thermodynamics at play when the chiral coupling is switched on.

In particular, the escape rates $\kappa=1 /\langle\tau\rangle$ corresponding to each of the three optical landscapes can be extracted from the measurements of the average residency times using the Poisson statistics. Since we show that our pseudo-potential approach allows us to predict very precisely the distribution of positions and since the optical landscapes can each be set very precisely, it thus becomes possible to perform an absolute determination of (i) $\operatorname{Re}[\chi]$ by measuring $\left\langle\tau^{\text {achiral }}\right\rangle /$ $\left\langle\tau_{A, C}^{\text {reac }}\right\rangle$ [see Eqs. (19) and (20)] and of (ii) $\operatorname{Im}[\chi]$ by measuring $\left\langle\tau^{\text {achiral }}\right\rangle /\left\langle\tau_{A, C}^{\text {diss }}\right\rangle$ [see Eqs. (24) and (25)]. This determination is done at the single-nanoparticle level, and as such draws promising detection capacities in the context of artificial chiral matter engineering at the nanoscale [63-66].

\section{CONCLUSION}

We study, in the framework of the Fokker-Planck equation, the stochastic motion of an overdamped Brownian chiral probe optically trapped, diffusing in a bistable potential energy landscape formed in the standing wave of two counterpropagating Gaussian beams. We analyze in this framework the modifications of the escape rates when a chiral coupling is induced between the probe and the optical field. We summarize the main results.

(i) The chiral coupling mediated by optical forces can be switched on inside the optical trap simply by selecting the polarizations of the counterpropagating beams forming the initial, achiral, bistable potential, while keeping the energy densities fixed.

(ii) Chiral coupling (of reactive and/or dissipative nature) leads to modifications of the thermodynamics of the thermal activation of the barrier that are enantiospecific and dependent on the enantiomorphic configurations of the chiral optical environment.

(iii) More precisely, reactive coupling takes the form of conservative chiral optical forces and thus contributes as an additional free-energy term to the potential energy of the bistable trap. The modifications of the free-energy landscape either strengthen the trapping potential or decreases the barrier height of the 
chirally dressed potential. These modifications can be swapped by changing the enantiomer within a fixed chirality of the optical environment or the optical enantiomorph for a chosen nanoparticle enantiomer.

(iv) The dissipative coupling yields nonconservative chiral forces that modify the thermal activation thermodynamics. In this nonequilibrium steady state of the system, the dissipation of heat to the thermal bath is responsible for lifting the degeneracy of the probability density function between the two local minima of the bistable potential. This breaking of the initial mirror symmetry of the bistable trap takes the form of an enantiospecific contribution to the thermodynamics.

(v) The contribution of both types of coupling to the global thermodynamics is also observed at the level of stochastic simulations of the Langevin equation for trajectory ensembles in the presence of external chiral forces. The simulations clearly show, in particular, the chiral discriminatory nature of the dissipative coupling that constitutes an explicit example of a deracemization process analyzed from the thermodynamics viewpoint.

(vi) At the level of Langevin dynamics of single diffusing trajectories thermally activated over the barrier separating the two wells, the same results are reached by measuring the Poisson statistics of the residency times for each local minima of the bistable potential without and with chiral coupling. Measuring a difference in the average residency times in the case of the dissipative chiral coupling demonstrates from the single-trajectory viewpoint the optomechanical deracemization process.

(vii) Approaching the problem from the residency time point of view shows how one can probe the thermodynamics of the system from time measurement sequences only, rather than from more demanding force measurements, and how one can obtain an absolute measurement of both the real and imaginary parts of the chiral polarizability of a single nanoparticle by extracting from the Poisson statistics the average residency times in the achiral, chiral reactive, and chiral dissipative coupling schemes.

Overall, our results illustrate how the chiral coupling transforms chiral degrees of freedom into true thermodynamic control parameters. They open a rich playground to further explore chiral light-matter interactions. The capacity of our model to solve the stochastic chiral bistable problem convinces us that the optical forces and residency times approaches can offer new and relevant insights into the thermodynamics of chiral systems immersed within chiral environments. Considering the ubiquity of such bistable landscapes in the realm of chirality, our model and our methods have a heuristic value that unfolds at the crossroad of chemistry and physics. In particular, at the quantum level, further extending our results to chiral quantum optics $[67,68]$ will give the possibility to study how chirality can impact quantum stochastic thermodynamics $[69,70]$. This opens up new perspectives yet to be explored.

\section{ACKNOWLEDGMENTS}

We thank J. Crassous, J.-P. Dutasta, T. W. Ebbesen, M. W. Hosseini, Ph. Lesot, and J.-P. Sauvage for discussions. This work of the Interdisciplinary Thematic Institute QMat, as part of the ITI 20212028 program of the University of Strasbourg, CNRS, and Inserm, was supported by IdEx Unistra (ANR 10 IDEX 0002), by SFRI STRAT'US Project (ANR 20 SFRI 0012), by the Labex NIE (ANR-11- LABX$0058 \mathrm{NIE}$ ) and CSC (ANR-10-LABX-0026 CSC) projects, and by the University of Strasbourg Institute for Advanced Study USIAS (ANR-10-IDEX-0002-02) under the framework of the French Investments for the Future Program.

\section{APPENDIX A: THE DUAL-BEAM \\ OPTICAL TRAP: OPTICAL LANDSCAPES AND OPTICAL FORCES}

We extend here the simplified discussions of Secs. III and IV in order to include magnetic force components and thus present the complete chiral force model in the dipolar regime [25]. We remind that our configuration consists of counterpropagating Gaussian beams identical in terms of intensity and spatial profile. In the paraxial approximation, this implies the cancellation of the Poynting vector $\Pi(\mathbf{r})$, both its orbital and spin components. From a force viewpoint, this implies the absence of any radiation pressure force field.

The polarization vectors $\mathbf{e}_{ \pm}$associated with each beam can be described in a generic way with

$$
\mathbf{e}_{+}=\left(\sqrt{1-h_{+}} \mathbf{e}_{l}+\sqrt{1+h_{+}} \mathbf{e}_{r}\right) / \sqrt{2}
$$

for the beam propagating along the $z>0$ direction and

$$
\mathbf{e}_{-}=\left(\sqrt{1-h_{-}} e^{i(\delta-\delta \theta)} \mathbf{e}_{l}+\sqrt{1+h_{-}} e^{i(\delta+\delta \theta)} \mathbf{e}_{r}\right) / \sqrt{2}
$$

for the counterpropagating beam ( $z<0$ direction). In order to understand the role of the different polarization setting parameters, we stress that $h_{+}$controls the helicity of the $z>0$ beam with $\mathbf{e}_{+}$varying from $\mathbf{e}_{l}$ when $h_{+}=-1$ to $\left(\mathbf{e}_{l}+\mathbf{e}_{r}\right) / \sqrt{2}$ (i.e., linear state of polarization) when $h_{+}=0$, and to $\mathbf{e}_{r}$ when $h_{+}=+1$. The main polarization axis of the beam remains arbitrarily fixed and constitutes a degree of freedom for the axisymmetric system. The phase at time $t=0$ is fixed as well, the system being invariant by translation of the initial time. For the counterpropagating beam, $\mathbf{e}_{-}$on the other hand, varies from $e^{i(\delta-\delta \theta)} \mathbf{e}_{l}$ when $h_{-}=-1$ to $e^{i \delta}\left(e^{-i \delta \theta} \mathbf{e}_{l}+e^{i \delta \theta} \mathbf{e}_{r}\right) / \sqrt{2}$ when $h_{-}=0$, and to $e^{i(\delta+\delta \theta)} \mathbf{e}_{r}$ when $h_{-}=+1$. As can be seen, the effect of the 
parameter $\delta$ is, in all cases, a global phase shift. It thus controls the relative phase between the counterpropagating beams. The $\delta \theta$ parameter rotates the polarization axis, as can be most clearly seen by decomposing the circular polarization vectors in the linear polarization basis. These two parameters $\delta$ and $\delta \theta$ are expressed in radians, either as a phase angle or as a physical angle between the main axes.

Extending Sec. IV to the magnetic case, we define the electric $W_{E}(\mathbf{r})=\varepsilon_{f} \mathbf{E}_{\mathrm{SW}} \cdot \mathbf{E}_{\mathrm{SW}}^{*} / 4$ and magnetic $W_{H}(\mathbf{r})=$ $\mu_{f} \mathbf{H}_{\mathrm{SW}} \cdot \mathbf{H}_{\mathrm{SW}}^{*} / 4$ components of the time-averaged energy density which can be expressed in terms of the trapping energy density $W_{\text {trap }}(\mathbf{r})$ and an interference energy density $W_{\text {inter }}(\mathbf{r})$ as

$$
\begin{aligned}
& W_{E}(\mathbf{r})=W_{\text {trap }}(\mathbf{r})+W_{\text {inter }}(\mathbf{r}), \\
& W_{H}(\mathbf{r})=W_{\text {trap }}(\mathbf{r})-W_{\text {inter }}(\mathbf{r}),
\end{aligned}
$$

where we have

$$
\begin{gathered}
W_{\text {trap }}(\mathbf{r})=\frac{\mathcal{E}_{0}^{2} w_{0}^{2} \varepsilon_{f}}{2 w^{2}(z)} e^{-\frac{2 q^{2}}{w^{2}(z)}}, \\
W_{\text {inter }}(\mathbf{r})=W_{\text {trap }}(\mathbf{r})\left[h_{2} \cos \delta \theta \cos \varphi(\mathbf{r})\right. \\
\left.+h_{1} \sin \delta \theta \sin \varphi(\mathbf{r})\right] .
\end{gathered}
$$

We also define the electric and magnetic ellipticities $\boldsymbol{\Phi}_{E}(\mathbf{r})=i \omega \varepsilon_{f} \mathbf{E}_{\mathrm{SW}} \times \mathbf{E}_{\mathrm{SW}}^{*} / 4$ and $\boldsymbol{\Phi}_{H}(\mathbf{r})=i \omega \mu_{f} \mathbf{H}_{\mathrm{SW}} \times$ $\mathbf{H}_{\mathrm{SW}}^{*} / 4$ that can be summed to obtain the chiral flux introduced Sec. V, $\boldsymbol{\Phi}(\mathbf{r})=\boldsymbol{\Phi}_{E}(\mathbf{r})+\boldsymbol{\Phi}_{H}(\mathbf{r}) \quad[25,32]$. These vectorial ellipticities decompose into average $\boldsymbol{\Phi}_{\text {trap }}(\mathbf{r})$ and interference $\boldsymbol{\Phi}_{\text {inter }}(\mathbf{r})$ flux components. This time, however, both components depend on the polarization of the beams according to

$$
\begin{gathered}
\boldsymbol{\Phi}_{E}(\mathbf{r})=\boldsymbol{\Phi}_{\text {trap }}(\mathbf{r})+\boldsymbol{\Phi}_{\text {inter }}(\mathbf{r}), \\
\boldsymbol{\Phi}_{H}(\mathbf{r})=\boldsymbol{\Phi}_{\text {trap }}(\mathbf{r})-\boldsymbol{\Phi}_{\text {inter }}(\mathbf{r}), \\
\boldsymbol{\Phi}(\mathbf{r})=2 \boldsymbol{\Phi}_{\text {trap }}(\mathbf{r}),
\end{gathered}
$$

with

$$
\begin{gathered}
\boldsymbol{\Phi}_{\text {trap }}(\mathbf{r})=-\omega \frac{h_{+}+h_{-}}{2} W_{\text {trap }}(\mathbf{r}) \mathbf{z}, \\
\boldsymbol{\Phi}_{\text {inter }}(\mathbf{r})=\omega W_{\text {trap }}(\mathbf{r})\left[h_{1} \cos \delta \theta \cos \varphi(\mathbf{r})\right. \\
\left.+h_{2} \sin \delta \theta \sin \varphi(\mathbf{r})\right] \mathbf{z} .
\end{gathered}
$$

Finally, we remind of the expression for the chiral density $K(\mathbf{r})=\omega \varepsilon_{f} \mu_{f} \operatorname{Im}\left[\mathbf{E}_{\mathrm{SW}} \cdot \mathbf{H}_{\mathrm{SW}}^{*}\right] / 2$, which corresponds to a simple trapping pattern modulated by the relative chirality of the beams

$$
K(\mathbf{r})=-\left(h_{+}-h_{-}\right) \times \omega \sqrt{\varepsilon_{f} \mu_{f}} W_{\text {trap }}(\mathbf{r}) .
$$

These fluxes and potentials allow us to fully define the electric, magnetic, and chiral forces

$$
\begin{aligned}
& \mathbf{F}_{E}^{\text {reac }}(\mathbf{r})=\operatorname{Re}[\alpha] \nabla W_{E}(\mathbf{r}), \\
& \mathbf{F}_{H}^{\text {reac }}(\mathbf{r})=\operatorname{Re}[\beta] \nabla W_{H}(\mathbf{r}), \\
& \mathbf{F}_{\chi}^{\text {reac }}(\mathbf{r})=\operatorname{Re}[\chi] \frac{\nabla K(\mathbf{r})}{\omega \sqrt{\varepsilon_{f} \mu_{f}}}, \\
& \mathbf{F}_{E}^{\text {diss }}(\mathbf{r})=\operatorname{Im}[\alpha]\left(\omega \varepsilon_{f} \mu_{f} \Pi(\mathbf{r})-\frac{\nabla \times \boldsymbol{\Phi}_{E}(\mathbf{r})}{\omega}\right), \\
& \mathbf{F}_{H}^{\text {diss }}(\mathbf{r})=\operatorname{Im}[\beta]\left(\omega \varepsilon_{f} \mu_{f} \boldsymbol{\Pi}(\mathbf{r})-\frac{\nabla \times \boldsymbol{\Phi}_{H}(\mathbf{r})}{\omega}\right), \\
& \mathbf{F}_{\chi}^{\text {diss }}(\mathbf{r})=\sqrt{\varepsilon_{f} \mu_{f}} \operatorname{Im}[\chi](2 \mathbf{\Phi}(\mathbf{r})-\nabla \times \mathbf{\Pi}(\mathbf{r}))
\end{aligned}
$$

that connect the real and imaginary parts of the electric magnetic polarizabilities $\alpha, \beta$, and $\chi$ to the electric, magnetic, and chiral densities and fluxes of the electromagnetic field, respectively.

In our configuration, the electric and magnetic dissipative forces are purely azimuthal, thus playing no role in the probability distributions of the double well. For our onoptical axis model, we can thus ignore them in the FokkerPlanck analysis where the only dissipative force that must be accounted for is the chiral dissipative force. Of course, these azimuthal components are accounted for in the threedimensional simulations of the vectorial Langevin equation.

\section{APPENDIX B: DIPOLAR CHIRAL NANOPARTICLE MODEL}

Here, we follow Ref. [32] in order to calculate the dipolar $(\mathbf{p}, \mathbf{m})$ response of a chiral nanosphere that can be expressed in terms of the incident electric and magnetic fields $\mathbf{E}$ and $\mathbf{H}$ :

$$
\left(\begin{array}{c}
\mathbf{p} \\
\mathbf{m}
\end{array}\right)=\left(\begin{array}{cc}
\alpha \varepsilon_{f} & i \chi \sqrt{\varepsilon_{f} \mu_{f}} \\
-i \chi \sqrt{\varepsilon_{f} / \mu_{f}} & \beta
\end{array}\right)\left(\begin{array}{l}
\mathbf{E} \\
\mathbf{H}
\end{array}\right) .
$$

In the quasistatic limit for a sphere of radius $R$, the electric, magnetic, and chiral susceptibilities $\alpha, \beta$, and $\chi$ are given as

$$
\begin{gathered}
\alpha=4 \pi R^{3} \frac{\left(\epsilon_{m}-\epsilon_{f}\right)\left(\mu_{m}+2 \mu_{f}\right)-\kappa_{m}^{2}}{\left(\epsilon_{m}+2 \epsilon_{f}\right)\left(\mu_{m}+2 \mu_{f}\right)-\kappa_{m}^{2}}, \\
\beta=4 \pi R^{3} \frac{\left(\epsilon_{m}+2 \epsilon_{f}\right)\left(\mu_{m}-\mu_{f}\right)-\kappa_{m}^{2}}{\left(\epsilon_{m}+2 \epsilon_{f}\right)\left(\mu_{m}+2 \mu_{f}\right)-\kappa_{m}^{2}}, \\
\chi=12 \pi R^{3} \frac{\kappa_{m}}{\left(\epsilon_{m}+2 \epsilon_{f}\right)\left(\mu_{m}+2 \mu_{f}\right)-\kappa_{m}^{2}},
\end{gathered}
$$

where $\epsilon_{m}$ and $\mu_{m}$ are the complex permittivity and permeability of the material (in our case, gold), $\epsilon_{f}$ and 
$\mu_{f}$ are those of the fluid (assuming that both are purely real), and $\kappa_{m}$ is the complex "chiral parameter" of the nanosphere [32].

In practice, for a nonspherical chiral particle of arbitrary geometry, it is reasonable to assume that these equations will still apply with an effective electromagnetic radius $R$ and a chiral parameter that depends on the geometry of the particle. Exact equations can, however, always be calculated knowing the shape of the particle. Experimentally, a determination of the complex chiral parameter can be obtained by measuring for the chiral nanoparticle the optical rotatory dispersion for $\operatorname{Re}\left[\kappa_{m}\right]$ and the circular dichroism for $\operatorname{Im}\left[\kappa_{m}\right]$.

From the chiral optical force perspectives, the polarizability $\chi$ is the relevant parameter, more precisely, the ratio $\xi=\chi / \alpha$ that we fix at a $5 \%$ value throughout the paper. The Clausius-Mossotti relations (B4) then lead to a relation that allows us to determine $\kappa_{m}$ from the chosen value for $\xi$ :

$$
\kappa_{m}^{2}+3 \kappa_{m} / \xi-\left(\epsilon_{m}-\epsilon_{f}\right)\left(\mu_{m}+2 \mu_{f}\right)=0
$$

Among the four possible solution values for $\kappa_{m}$, we use the two opposite ones that have the smallest modulus. This choice is made in order to ensure that the transition from the achiral $\chi=0$ case to the chiral $\chi \neq 0$ case has practically no impact on the $\alpha$ and $\beta$ values. In such a case, the trapping potential profile described in Sec. IV in the achiral case remains unchanged when the chiral coupling is induced in Secs. VI A and VI B with $\chi \neq 0$ and nonzero chiral density and/or flux.

\section{APPENDIX C: ACHIRAL FORCE-FIELD LANDSCAPE}

Here we describe the general polarization parameter space in which the achiral electric force-field landscape develops, as illustrated in Fig. 10. By choosing the $h_{+}, h_{-}$, and $\delta \theta$ parameters, we can tune the relative amplitude of the interference $\operatorname{Re}[\alpha] \nabla W_{\text {inter }}$ (blue line of the insets) and average trapping forces $\operatorname{Re}[\alpha] \nabla W_{\text {trap }}$ (black line of the insets of Fig. 10), while the $\delta$ parameters introduces a phase change in the interference forces. In Fig. 10, as well as in
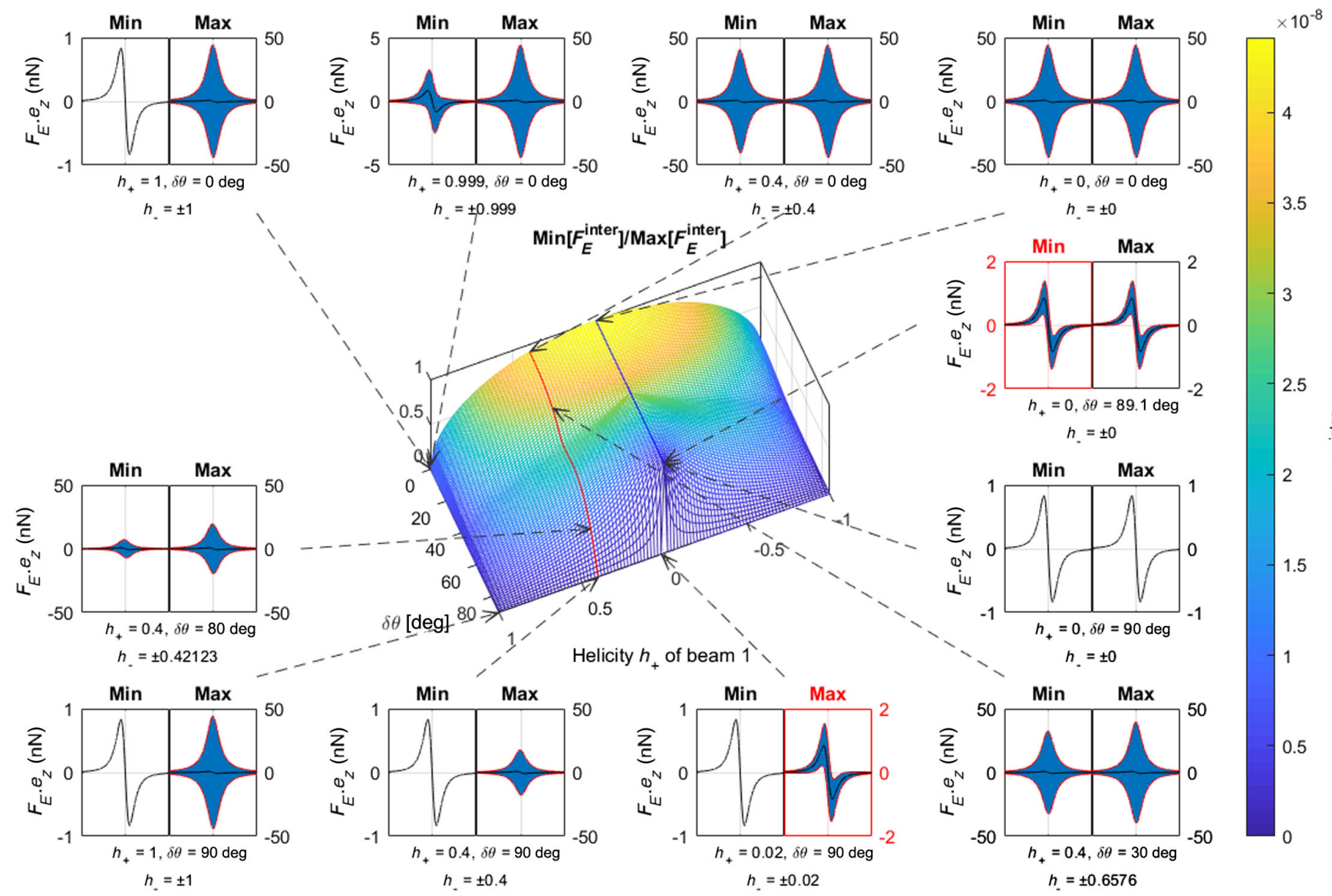

FIG. 10. General $\left(h_{+}, h_{-}, \delta, \delta \theta\right)$ polarization parameter space describing the achiral electric force-field landscape whose equations are detailed in Appendix A in terms of the ratio between the minimum and the maximum amplitude of the interference forces. All insets display forces along the optical axis from both sides of the waist over a length of $20 \mu \mathrm{m}$. Note that the force amplitudes of the insets vary from configuration to configuration. 
the rest of the paper, we systematically choose $\delta$ so that the interference potential is maximum at the center of the trap $(z=0)$. The surface represents the ratio between the maximum and the minimum amplitude of the interference forces when varying $h_{-}$against the choice of $h_{+}$and $\delta \theta$.

The insets displayed in the figure showcase a few archetypical configurations. For a given choice of $h_{+}$ and $\delta \theta$, we present the choices of $h_{-}$where the amplitude of the interference forces are, respectively, minimum and maximum. These two configurations are set with two opposite values of $h_{-}$that are specified in the insets. The oscillations of the interference force in blue appear as a blue surface due to the high frequency of the oscillations with respect to the extension chosen for the optical axis. The red lines in the insets are the envelope of the total force. If we vary $\delta \theta$ along the $h_{+}=0$ line (in blue on the surface), the intensity of the interferences is varied but does not change depending on $h_{-}$. The achiral landscape used in the main text is a more extreme case of the inset outlined in red on the right. It is shown in detail in Fig. 11 below. The other red outlined inset is a reactive configuration-as the maximum of interference is obtained for opposite values of $h_{ \pm}-$close to the one chosen in the main text.

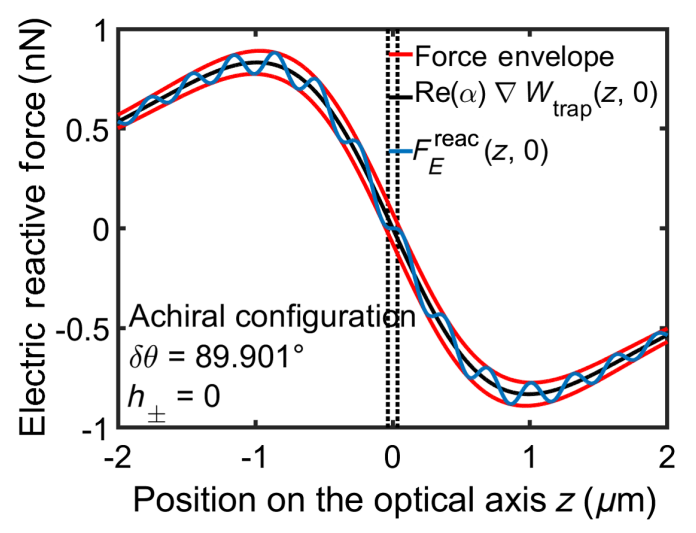

FIG. 11. Enlargement of the electric force landscape in the achiral case, keeping in mind that the magnetic force is practically zero. This landscape is modified by the chiral forces when the helicity parameters $h_{ \pm}$are nonzero. As in Fig. 10, the black line represents the force deriving from the trapping potential $-\operatorname{Re}[\alpha] \nabla W_{\text {trap }}(z, q)$. This force carries the contribution of the interference potential $-\operatorname{Re}[\alpha] \nabla W_{\text {inter }}(z, q)$ due to the interplay between the two counterpropagating beams. The total reactive electric force generated by these two components is represented by the blue line. Finally, the red line is the envelope of the electric forces. In the main text, we consider the nanospheres trapped at the center of the well at $z=0$, with Fig. 3(b) displaying the force landscape evaluated between the dotted black lines. There is locally a double well caused by the oscillation due to the interference force around $z=0$. In this configuration, we have only two wells, due to the fact that the interference forces are not strong enough to generate other trapping locations, as can be seen by the fact that they do not cross 0 except at the center, and this despite their varying intensity.
The dissipative configuration is an intermediate case where $\delta \theta \neq 90^{\circ}$ allows for a purely dissipative interference force landscape. Along the red line on the surface, we vary $\delta \theta$ from $0^{\circ}$ to $90^{\circ}$, allowing for such intermediate cases to appear. Finally, in the configurations where $\delta \theta=90^{\circ}$ or $h_{+}=1$, we ensure that the minimum of interferences is always 0 while for $\delta \theta=0^{\circ}$, we ensure that the maximum of interference is the global maximum. For other intermediate values of $\delta \theta$, the choice of $h_{+}$can tune the minimum of interferences, ensuring that they are present, as seen, for example, following the red line.

\section{APPENDIX D: FOKKER-PLANCK MODEL FOR THERMAL ACTIVATION OF A BARRIER CROSSING: BISTABLE EQUILIBRIUM}

We describe here the thermodynamic evolution of a dipole inside a bistable potential at temperature $T$, basing our description within the classical framework proposed by Kramers [41]. Within this framework and without chiral contributions $[K(\mathbf{r})=0, \boldsymbol{\Phi}(\mathbf{r})=\mathbf{0}]$, we describe the evolution of the dipole inside the bistable potential as being driven only by decoupled achiral reactive axial $\mathbf{F}_{\alpha}(\mathbf{r}) \cdot \hat{\mathbf{z}}$ and radial $\mathbf{F}_{\alpha}(\mathbf{r}) \cdot \hat{\boldsymbol{\rho}}$ forces inside the optical trap. This situation corresponds to a Kramers problem with the possibility given to the dipole to escape local trapping sites by thermal activation and diffusion over the separating barrier of the double-well potential energy landscape drawn in Fig. 3 in the main text. We model this metastable dynamics with an overdamped Fokker-Planck equation [48]

$$
\partial_{t} p(\mathbf{r}, t)=-\boldsymbol{\nabla} \cdot \mathbf{j}(\mathbf{r}, t)
$$

connecting the probability density $p(\mathbf{r}, t)$ to find the dipole at $\mathbf{r}$ at time $t$ to the probability current

$$
\mathbf{j}(\mathbf{r}, t)=-\frac{1}{\gamma} \boldsymbol{\nabla} U_{\mathrm{opt}}(\mathbf{r}) p(\mathbf{r}, t)-D \boldsymbol{\nabla} p(\mathbf{r}, t),
$$

with $\gamma$ the Stokes drag coefficient, $k_{B}$ the Boltzmann constant, and $D=k_{B} T / \gamma$ the free Brownian diffusion coefficient. For an optical trap immobilizing an Au nanosphere of radius $R=20 \mathrm{~nm}$ in pure water at room temperature $\left(\gamma=2 \pi \eta R\right.$ with a viscosity $\left.\eta=0.88 \times 10^{-3} \mathrm{Kg} / \mathrm{m} / \mathrm{s}\right)$, the overdamped regime is well reached with a momentum relaxation time given by the nanoparticle-over-friction ratio of $m / \gamma \sim 10^{-7}$ s.

In the model developed in the main text, we study only the component $j_{z}(\mathbf{r}, t)$ of the probability current along the optical $z$ axis in the steady-state regime with $U_{\text {opt }}$ time independent. In this regime, $\partial_{t} p(\mathbf{r}, t)=0$ implies that

$$
\frac{1}{q} \partial_{q}\left(q j_{\rho}(\mathbf{r}, t)\right)+\partial_{z} j_{z}(\mathbf{r}, t)=0 .
$$

We now further neglect the transverse variations of the beam with respect to the axial ones, i.e., $\partial_{q}\left(q j_{\rho}\right) / q \ll \partial_{z} j_{z}$, 
so that the $z$ component of the probability current around the waist is modeled as a constant $j_{z}(q)$ in the $z$ variable. This makes it easy to evaluate the crossing rates for the dipole over the barrier (positioned at $z_{B}=0$ ) in the forward + and backward $-z$ directions. In the forward $z$ direction, the rate to be computed corresponds to crossing events from the $z<0$ initially populated well (well $A$, minimum at $z_{A}$ ) toward the $z>0$ unoccupied one (well $C$, minimum at $z_{C}$ ). This initial population corresponds to the stationary nonequilibrium probability density $p^{+}(q, z)$ inside well $A$ that creates a current $j_{z}^{+}(q)=\mathbf{j}(q) \cdot \hat{\mathbf{z}}$ flowing in the $+z$ direction according to

$$
\begin{aligned}
j_{z}^{+}(q)= & -\frac{1}{\gamma} \partial_{z} U_{\mathrm{opt}}(q, z) p^{+}(q, z) \\
& -D \partial_{z} p^{+}(q, z)
\end{aligned}
$$

Following a standard method [71], the nonequilibrium probability density $p^{+}(q, z)$ can be determined between one point $z^{\prime}$ within well $A$ and a distant point above the barrier for which $p^{+}\left(q, z^{+}>z_{B}\right)=0$ as

$$
p^{+}\left(q, z^{\prime}\right)=\gamma j_{z}^{+}(q) e^{\frac{-U_{\mathrm{opt}}\left(q, z^{\prime}\right)}{k_{B} T}} \int_{z^{\prime}}^{z^{+}} d u e^{\frac{U_{\mathrm{opt}}(q, u)}{k_{B} T}}
$$

together with the corresponding population density

$$
n_{A}^{+}=\int_{0}^{+\infty} d q 2 \pi q \int_{-\infty}^{z_{B}} d z^{\prime} p^{+}\left(q, z^{\prime}\right)
$$

This population is then evaluated with a Gaussian steepest-descent approximation, expanding the optical potential around the barrier maximum at $z_{B}$ and the local minimum at $z_{A}$ :

$$
\begin{aligned}
& U_{\mathrm{opt}}\left(q, z \sim z_{B}\right) \simeq U_{\mathrm{opt}}\left(q, z_{B}\right)-b^{2}(q)\left(z-z_{B}\right)^{2}, \\
& U_{\mathrm{opt}}\left(q, z \sim z_{A}\right) \simeq U_{\mathrm{opt}}\left(q, z_{A}\right)+a^{2}(q)\left(z-z_{A}\right)^{2},
\end{aligned}
$$

with $2 b^{2}(q)=\left|\partial^{2} U_{\text {opt }}(q, z) / \partial z^{2}\right|_{z_{B}} \quad$ and $\quad 2 a^{2}(q)=$ $\left|\partial^{2} U_{\text {opt }}(q, z) / \partial z^{2}\right|_{z_{A}}$, and extending the lower and upper limits of integration to $\pm \infty$. We thus obtain

$$
n_{A}^{+} \simeq \int_{0}^{+\infty} d q 2 \pi q \frac{k_{B} T \gamma \pi j_{z}^{+}(q)}{a(q) b(q)} e^{\left[U_{\mathrm{opt}}\left(q, z_{B}\right)-U_{\mathrm{opt}}\left(q, z_{A}\right) / k_{B} T\right]} .
$$

Because of the axial symmetry of the optical landscape, it is clear that the optical potential is an even function of $q$. Therefore, in close vicinity to the optical axis, one can always assume that $\left(\partial^{2} U_{\text {opt }} / \partial q \partial z\right)(q, z) \sim 0$. This assumption has two consequences: (i) that $a(q) \sim a$ and $b(q) \sim b$ are independent of $q$, and (ii) that $U_{\text {opt }}\left(q, z_{B}\right)-$ $U_{\text {opt }}\left(q, z_{A}\right) \simeq U_{\text {opt }}\left(0, z_{B}\right)-U_{\text {opt }}\left(0, z_{A}\right)$ by expanding the potential energy around $z_{A}$ and $z_{B}$. Therefore, under this hypothesis,

$n_{A}^{+} \simeq \frac{k_{B} T \gamma \pi}{a b} e^{\left[U_{\mathrm{opt}}\left(0, z_{B}\right)-U_{\mathrm{opt}}\left(0, z_{A}\right) / k_{B} T\right]} \int_{0}^{+\infty} d q 2 \pi q j_{z}^{+}(q)$.

The assumption leads us to interpret $\int_{0}^{+\infty} d q 2 \pi q j_{z}^{+}(q)=$ $J_{z}^{+}$as the total probability current in the positive direction. The escape rate $\kappa_{A \rightarrow C}$ from well $A$ to well $C$ then simply writes as

$$
\kappa_{A \rightarrow C}=\frac{J_{z}^{+}}{n_{A}^{+}} \simeq \frac{a b}{k_{B} T \gamma \pi} e^{-\left[U_{\mathrm{opt}}\left(0, z_{B}\right)-U_{\mathrm{opt}}\left(0, z_{A}\right) / k_{B} T\right]},
$$

which corresponds to the well-known result obtained by Kramers with $\Delta U_{\text {opt }}^{A B}=U_{\text {opt }}\left(0, z_{B}\right)-U_{\text {opt }}\left(0, z_{A}\right)$ the optical barrier height measured along the optical axis at $q=0$ $[41,48]$.

The escape rate $\kappa_{C \rightarrow A}$ from well $C$ to well $A$ is calculated from the probability current $j_{z}^{-}(q)=\mathbf{j}^{-}(q) \cdot(-\mathbf{z})$ flowing in the opposite direction of $j_{z}^{+}(q)$ and solution of

$$
\begin{aligned}
j_{z}^{-}(q)= & +\frac{1}{\gamma} \partial_{z} U_{\mathrm{opt}}(q, z) p^{-}(q, z) \\
& +D \partial_{z} p^{-}(q, z)
\end{aligned}
$$

Following the same steps, but this time integrating over well $C$, one evaluates the escape rate from well $C$ at $z_{C}$ over the barrier at $z_{B}$ as

$$
\kappa_{C \rightarrow A}=\frac{J_{z}^{-}}{n_{C}^{-}} \simeq \frac{b c}{k_{B} T \gamma \pi} e^{-\left[U_{\mathrm{opt}}\left(0, z_{B}\right)-U_{\mathrm{opt}}\left(0, z_{C}\right) / k_{B} T\right]} .
$$

For a symmetric optical potential with $U_{\text {opt }}\left(0, z_{A}\right)=$ $U_{\text {opt }}\left(0, z_{C}\right)$ and $a=c$, one obviously obtains $n_{A}^{+}=n_{C}^{-}$and therefore from the detailed balance $\kappa_{A \rightarrow C}=\kappa_{C \rightarrow A}$. We can take this equality and the absence of any other force besides the trapping force forming the optical bistable potential energy as the definition of the equilibrium state of our system.

\section{APPENDIX E: SIMULATIONS: ALGORITHMS AND METHODS}

The Langevin dynamics of an overdamped Brownian object at position $\mathbf{r}$ immersed in a force field $\mathbf{F}$ and a fluid Stokes drag coefficient $\gamma$ and diffusion coefficient $D$ is given by the equation

$$
d \mathbf{r}=\frac{1}{\gamma} \mathbf{F} d t+\sqrt{2 D} d \mathbf{W}_{t}
$$

where $d \mathbf{W}_{t}$ is the Brownian increment at time $t$. 
To simulate the Langevin dynamics of a dipolar chiral particle in an axisymmetrical force field as done in Secs. VII and VIII, we use the Euler-Maruyama scheme [72]

$$
\begin{aligned}
\rho_{n}= & \sqrt{x_{n}^{2}+y_{n}^{2}}, \\
x_{n+1}= & x_{n}+\frac{d t}{\gamma} \frac{x_{n} F_{\rho}\left(\rho_{n}, z_{n}\right)-y_{n} F_{\theta}\left(\rho_{n}, z_{n}\right)}{\rho_{n}} \\
& +\sqrt{2 D d t} \times \eta_{x}(n), \\
y_{n+1}= & y_{n}+\frac{d t}{\gamma} \frac{y_{n} F_{\rho}\left(\rho_{n}, z_{n}\right)+x_{n} F_{\theta}\left(\rho_{n}, z_{n}\right)}{\rho_{n}} \\
& +\sqrt{2 D d t} \times \eta_{y}(n), \\
z_{n+1}= & z_{n}+\frac{d t}{\gamma} F_{z}\left(\rho_{n}, z_{n}\right)+\sqrt{2 D d t} \times \eta_{z}(n),
\end{aligned}
$$

where during the time increment $d t$, the Brownian increment on each axis is randomly chosen in the distribution $\eta_{x / y / z}=\sqrt{d t} \mathcal{N}(0,1)$. The simulation time-step parameter $d t$ is chosen such that $\max \left[F_{i} \sqrt{d t} / \sqrt{2 k_{B} T \gamma}\right] \leq 1$ for all cylindrical components $i=\rho, \theta, z$ of the optical force $\mathbf{F}$ (achiral and chiral).

In an effort to further reduce the calculation time and thus allow for better statistics to be used, we use the result of our one-dimensional model and draw the initial positions from the predicted stationary PDF in order to avoid the equilibration time. To do that, we use a multidimensional inverse-transform sampling method.

In a standard one-dimensional inverse-transform sampling, knowing the distribution's PDF $p(X)$, we calculate the monotonic cumulative distribution function (CDF) $F(X)$. It can then be proved that if we draw a random number $U$ following a uniform distribution, $F^{-1}(U)$ will follow the distribution $p(X)$. In order to adapt this method to our multidimensional case, we first note that the problem being fully axisymmetrical, the azimuth $\theta$ can simply be chosen as a uniformly distributed random number. The two remaining parameters are then the axis and radius coordinates $z$ and $q$.

As in the one-dimensional method, we calculate the PDF $p(q, z)$ obtained using our pseudo-potential model described in Sec. VIB as $p(q, z)=C \exp [-\varphi(q, z)]$, with $\varphi(q, z)=\left[U_{\mathrm{opt}}(q, z)+u_{\chi}^{\mathrm{diss}}(q, z)\right] / k_{B} T$ and $C=$ $\int_{0}^{+\infty} \int_{-\infty}^{+\infty} \exp [-\varphi(q, z)] d z 2 \pi q d q$. Its $\operatorname{CDF} F(z, q)$ is defined by

$$
F(z, q)=\int_{-\infty}^{z} \int_{0}^{q} p\left(q^{\prime}, z^{\prime}\right) 2 \pi q^{\prime} d q^{\prime} d z^{\prime} .
$$

Since $p(q, z)$ has a complicated expression that cannot be easily inverted or integrated, we calculate $F(z, q)$ numerically over a large enough domain $\left[-z_{M} ; z_{M}\right]$ for $z$ and $\left[0 ; q_{M}\right]$ for $q$ and numerically perform the necessary inversions. We can then consider $F_{z}(z)=F(z,+\infty)$ and apply the inverse-transform sampling method using $F_{z}(z)$ to pick a random number $z_{c}$ following the distribution $p_{z}(z)=\int_{0}^{+\infty} p(q, z) 2 \pi q d q$. In this context, it means that picking a random number $\eta_{z}$ in the uniform distribution on [0;1[, we can find $z_{c}=F_{z}^{(-1)}\left(\eta_{z}\right)$. Finally, we define $\left.F_{q}\right|_{z=z_{c}}(q)=F\left(z_{c}, q\right) / F\left(z_{c},+\infty\right)$ and use again the inverse-transform sampling method to pick up a random number $q_{c}$ in the distribution $\left.p_{q}\right|_{z=z_{c}}(q)=p\left(q, z=z_{c}\right)$. To do that, we again pick up a random number $\eta_{q}$ uniformly distributed in $\left[0 ; 1\left[\right.\right.$ and apply $q_{c}=\left.F_{q}\right|_{z=z_{c}} ^{(-1)}\left(\eta_{q}\right)$. The pair $\left(q_{c}, z_{c}\right)$ of generated numbers thus follows the distribution $p(q, z)$.

Repeating this method for each trajectory, we generate the initial distribution for our simulation using the stationary predictions from our one-dimensional model PDF. If this distribution were not the stationary distribution, it would relax toward it in the course the simulation, leading to significant time spent in stabilizing the distribution rather than generating usable data. The one-dimensional model induces only errors small enough that the possible relaxation of the PDF parameters is dominated by their intrinsic thermal fluctuation. By generating a large number of steady-state trajectories, we can however check that using this distribution, the statistical parameters do not change in a measurable way over the simulated time. Therefore, all the generated time steps can be used for the data analysis of the properties of our simulated system in its steady state.

\section{APPENDIX F: RESIDENCE TIME PROBABILITY DENSITY FUNCTIONS}

Section VIII analyzes the distribution of the residency times in both wells $A$ and $C$ of the optical potential energy as a function of the presence and nature of the chiral coupling. These residence times are calculated using 4096 trajectories long of 3000000 steps. We describe in this section how the residency times are identified.

A diffusing trajectory in the bistable potential is characterized by different jumplike events. For some, the particle moves quickly from one well to the other. For many others, however, the particles diffuse around the top of the unstable barrier or barely cross it and return back to their initial wells, so-called recrossing events.

Following Ref. [73], we choose to use a hysteresis criterion to filter out such recrossing events. To do this, we exploit the repulsive character of the barrier whose strength is given by a steepest-descent approach similar to the one developed in Sec. VI as $-\left(\partial^{2} \varphi_{\text {eff }} / \partial z^{2}\right)$, where $\varphi_{\text {eff }}=U_{\text {opt }}+U_{\chi}^{\text {reac }}+u_{\chi}^{\text {diss }}$ is evaluated on the optical axis $(q=0, z)$ including the chiral reactive potential $U_{\chi}^{\text {reac }}$ and/or dissipative pseudo-potential $u_{\chi}^{\text {diss }}$ depending on the chiral-coupling cases. 
This trapping strength leads to a standard deviation delimiting an exclusion zone of $\sigma=\sqrt{-k_{B} T /\left(\partial^{2} \varphi_{\text {eff }} / \partial z^{2}\right)}=$ $10 \mathrm{~nm}$. We use this standard deviation to define the hysteresis of the bistability: The particle enters or leaves well $A$ when it crosses the $z=-\sigma$ and enters or leaves well $C$ when it crosses $z=\sigma$. But in addition, a jump is counted only when the opposite well is reached. In other words, a particle that would make an excursion in the vicinity of the barrier $z_{B}$ and eventually going back to its initial well will not be counted as having left its well. Such sequences are excluded from the record, as seen in black on Fig. 9 in the main text.

Having defined the crossing events, as shown in Figs. 9(a) and 9(c), we measure the time interval $\tau$ that a particle has stayed in one well before jumping to the other. Because it is impossible to determine this time interval at the beginning and end of the trajectory, the corresponding events are excluded from the analysis.

We then calculate the PDF of the occupation times of both wells. The results are shown in Figs. 9(b) and 9(d). According to Kramers theory, this PDF should follow an exponential law. However, we clearly observe deviations from such a law at short times, where the position of the particle remains correlated. The correlation time being $t_{\text {corr }}=2 \pi \gamma /\left|\partial^{2} \varphi_{\text {eff }} / \partial z^{2}\right|_{\left(0, z_{A / C}\right)}$, we therefore exclude from our analysis all traces recorded for times smaller that $t_{\text {corr }}$. This being done, we finally perform a weighted fit of the distribution to take into account the fact that the smaller the probability, the lower the signal-over-noise ratio. This fit yields precise values for the slopes of the exponential law plotted in a logarithmic plot as represented in Figs. 9(b) and 9(d). From the Poissonian exponential law, these slopes correspond to the average residence times.

[1] S.-H. Yang, Spintronics on Chiral Objects, Appl. Phys. Lett. 116, 120502 (2020).

[2] N. P. M. Huck, W. F. Jager, B. de Lange, and B. L. Feringa, Dynamic Control and Amplification of Molecular Chirality by Circularly Polarized Light, Science 273, 1686 (1996).

[3] R. D. Astumian, Thermodynamics and Kinetics of a Brownian Motor, Science 276, 917 (1997).

[4] M. Avalos, R. Babiano, P. Cintas, J. L. Jiménez, J. C. Palacios, and L. D. Barron, Absolute Asymmetric Synthesis under Physical Fields: Facts and Fictions, Chem. Rev. 98, 2391 (1998).

[5] U. Hananel, A. Ben-Moshe, D. Tal, and G. Markovich, Enantiomeric Control of Intrinsically Chiral Nanocrystals, Adv. Mater. 32, 1905594 (2020).

[6] M. L. Slkeczkowski, M. F. Mabesoone, P. Slkeczkowski, A. R. Palmans, and E. Meijer, Competition between Chiral Solvents and Chiral Monomers in the Helical Bias of Supramolecular Polymers, Nat. Chem. 13, 200 (2021).

[7] M. Sarfati, P. Lesot, D. Merlet, and J. Courtieu, Theoretical and Experimental Aspects of Enantiomeric Differentiation Using Natural Abundance Multinuclear NMR Spectroscopy in Chiral Polypeptide Liquid Crystals, Chem. Commun. 2000, 2069 (2000).

[8] P. Lesot, C. Aroulanda, H. Zimmermann, and Z. Luz, Enantiotopic Discrimination in the NMR Spectrum of Prochiral Solutes in Chiral Liquid Crystals, Chem. Soc. Rev. 44, 2330 (2015).

[9] P. Lesot, C. Aroulanda, P. Berdagu, A. Meddour, D. Merlet, J. Farjon, N. Giraud, and O. Lafon, Multinuclear NMR in Polypeptide Liquid Crystals: Three Fertile Decades of Methodological Developments and Analytical Challenges, Prog. Nucl. Magn. Reson. Spectrosc. 116, 85 (2020).

[10] T. Fornstedt, P. Sajonz, and G. Guiochon, Thermodynamic Study of an Unusual Chiral Separation. Propranolol Enantiomers on an Immobilized Cellulase, J. Am. Chem. Soc. 119, 1254 (1997).

[11] N. M. Maier, P. Franco, and W. Lindner, Separation of Enantiomers: Needs, Challenges, Perspectives, J. Chromatogr. A 906, 3 (2001).

[12] H. Kagan, A. Moradpour, J. F. Nicoud, G. Balavoine, R. Martin, and J. Cosyn, Photochemistry with Circularly Polarised Light. II) Symmetric Synthesis of Octa and Nonahelicene, Tetrahedron Lett. 12, 2479 (1971).

[13] Y. Inoue, Asymmetric Photochemical Reactions in Solution, Chem. Rev. 92, 741 (1992).

[14] B. L. Feringa and R. A. Van Delden, Absolute Asymmetric Synthesis: The Origin, Control, and Amplification of Chirality, Angew. Chem., Int. Ed. Engl. 38, 3418 (1999).

[15] W. A. Bonner, The Quest for Chirality, AIP Conf. Proc. 379, 17 (1996).

[16] W. A. Bonner, The Origin and Amplification of Biomolecular Chirality, Origins Life Evol. Biosphere 21, 59 (1991).

[17] J. L. Bada, Origins of Homochirality, Nature (London) 374, 594 (1995).

[18] J. S. Siegel, Homochiral Imperative of Molecular Evolution, Chirality 10, 24 (1998).

[19] D. K. Kondepudi and G.-W. Nelson, Chiral Symmetry Breaking in Nonequilibrium Systems, Phys. Rev. Lett. 50, 1023 (1983).

[20] D. G. Blackmond, The Origin of Biological Homochirality, Cold Spring Harbor Perspect. Biol. 2, a002147 (2010).

[21] D. K. Kondepudi and K. Asakura, Chiral Autocatalysis, Spontaneous Symmetry Breaking, and Stochastic Behavior, Acc. Chem. Res. 34, 946 (2001).

[22] M. Quack, J. Stohner, and M. Willeke, High-Resolution Spectroscopic Studies and Theory of Parity Violation in Chiral Molecules, Annu. Rev. Phys. Chem. 59, 741 (2008).

[23] B. Darquié, C. Stoeffler, A. Shelkovnikov, C. Daussy, A. Amy-Klein, C. Chardonnet, S. Zrig, L. Guy, J. Crassous, P. Soulard et al., Progress toward the First Observation of Parity Violation in Chiral Molecules by High-Resolution Laser Spectroscopy, Chirality 22, 870 (2010).

[24] J. Cronin and J. Reisse, Chirality and the Origin of Homochirality, in Lectures in Astrobiology, edited by M. Gargaud, B. Barbier, H. Martin, and J. Reisse (Springer Berlin Heidelberg, Berlin, Heidelberg, 2005), Vol. I, pp. 473-515, https://doi.org/10.1007/10913406_14.

[25] A. Canaguier-Durand, J. A. Hutchison, C. Genet, and T. W. Ebbesen, Mechanical Separation of Chiral Dipoles by Chiral Light, New J. Phys. 15, 123037 (2013). 
[26] R. P. Cameron, S. M. Barnett, and A. M. Yao, Discriminatory Optical Force for Chiral Molecules, New J. Phys. 16, 013020 (2014).

[27] K. Ding, J. Ng, L. Zhou, and C. T. Chan, Realization of Optical Pulling Forces Using Chirality, Phys. Rev. A 89, 063825 (2014).

[28] K. Y. Bliokh, Y. S. Kivshar, and F. Nori, Magnetoelectric Effects in Local Light-Matter Interactions, Phys. Rev. Lett. 113, 033601 (2014).

[29] G. Tkachenko and E. Brasselet, Optofluidic Sorting of Material Chirality by Chiral Light, Nat. Commun. 5, 3577 (2014).

[30] R. P. Cameron, A. M. Yao, and S. M. Barnett, Diffraction Gratings for Chiral Molecules and Their Applications, J. Phys. Chem. A 118, 3472 (2014).

[31] A. Canaguier-Durand and C. Genet, Chiral near Fields Generated from Plasmonic Optical Lattices, Phys. Rev. A 90, 023842 (2014).

[32] A. Canaguier-Durand and C. Genet, Chiral Route to Pulling Optical Forces and Left-Handed Optical Torques, Phys. Rev. A 92, 043823 (2015).

[33] A. Hayat, J. P. B. Mueller, and F. Capasso, Lateral Chirality-Sorting Optical Forces, Proc. Natl. Acad. Sci. U.S.A. 112, 13190 (2015).

[34] I. D. Rukhlenko, N. V. Tepliakov, A. S. Baimuratov, S. A. Andronaki, Y. K. Gunko, A. V. Baranov, and A. V. Fedorov, Completely Chiral Optical Force for Enantioseparation, Sci. Rep. 6, 36884 (2016).

[35] N. Kravets, A. Aleksanyan, H. Chraibi, J. Leng, and E. Brasselet, Optical Enantioseparation of Racemic Emulsions of Chiral Microparticles, Phys. Rev. Applied 11, 044025 (2019).

[36] V. Marichez, A. Tassoni, R. P. Cameron, S. M. Barnett, R. Eichhorn, C. Genet, and T. M. Hermans, Mechanical Chiral Resolution, Soft Matter 15, 4593 (2019).

[37] K. Sekimoto, Stochastic Energetics (Springer-Verlag, Berlin, 2010).

[38] U. Seifert, Stochastic Thermodynamics, Fluctuation Theorems and Molecular Machines, Rep. Prog. Phys. 75, 126001 (2012).

[39] S. Ciliberto, Experiments in Stochastic Thermodynamics: Short History and Perspectives, Phys. Rev. X 7, 021051 (2017).

[40] J. Bechhoefer, S. Ciliberto, S. Pigolotti, and E. Roldán, Stochastic Thermodynamics: Experiment and Theory, J. Stat. Mech. (2020) 064001.

[41] H. A. Kramers, Brownian Motion in a Field of Force and the Diffusion Model of Chemical Reactions, Physica (Utrecht) 7, 284 (1940).

[42] V. I. Mel'nikov, The Kramers Problem: Fifty Years of Development, Phys. Rep. 209, 1 (1991).

[43] J. Shao and P. Hänggi, Control of Molecular Chirality, J. Chem. Phys. 107, 9935 (1997).

[44] B. Peters, Reaction Rate Theory and Rare Events (Elsevier, New York, 2017).

[45] F. Hund, Zur deutung der molekelspektren. III., Z. Phys. 43, 805 (1927).

[46] L. D. Barron, True and False Chirality and Absolute Enantioselection, Rend. Fis. Acc. Lincei 24, 179 (2013).
[47] L. Pasteur, in Oeuvres de Pasteur, Tome I, réunies par Pasteur Vallery-Radot, Recherches sur la dissymétrie moléculaire des produits organiques naturels (Leçons professées à la Société chimique de Paris le 20 janvier et 3 février 1860), pp. 314-344, http://catalogue.bnf.fr/ark:/ $12148 / \mathrm{cb} 37416454 \mathrm{q}$.

[48] P. Hänggi, P. Talkner, and M. Borkovec, Reaction-Rate Theory: Fifty Years after Kramers, Rev. Mod. Phys. 62, 251 (1990).

[49] D. B. Amabilino and R. M. Kellogg, Spontaneous Deracemization, Isr. J. Chem. 51, 1034 (2011).

[50] A. R. A. Palmans, Deracemisations under Kinetic and Thermodynamic Control, Mol. Syst. Des. Eng. 2, 34 (2017).

[51] L. D. Barron, Molecular Light Scattering and Optical Activity (Cambridge University Press, Cambridge, England, 2004).

[52] S. Stenholm, The Semiclassical Theory of Laser Cooling, Rev. Mod. Phys. 58, 699 (1986).

[53] D. B. Ruffner and D. G. Grier, Comment on "Scattering Forces from the Curl of the Spin Angular Momentum of a Light Field, Phys. Rev. Lett. 111, 059301 (2013).

[54] A. Canaguier-Durand, A. Cuche, C. Genet, and T. W. Ebbesen, Force and Torque on an Electric Dipole by Spinning Light Fields, Phys. Rev. A 88, 033831 (2013).

[55] Y. Zhao, A. Saleh, M. Van de Haar, B. Baum, J. Briggs, A. Lay, O. Reyes-Becerra, and J. Dionne, Nanoscopic Control and Quantification of Enantioselective Optical Forces, Nat. Nanotechnol. 12, 1055 (2017).

[56] A. Ashkin, Acceleration and Trapping of Particles by Radiation Pressure, Phys. Rev. Lett. 24, 156 (1970).

[57] S. B. Smith, Y. Cui, and C. Bustamante, Overstretching BDNA: The Elastic Response of Individual Double-Stranded and Single-Stranded DNA Molecules, Science 271, 795 (1996).

[58] A. van der Horst, P. D. J. van Oostrum, A. Moroz, A. van Blaaderen, and M. Dogterom, High Trapping Forces for High-Refractive Index Particles Trapped in Dynamic Arrays of Counterpropagating Optical Tweezers, Appl. Opt. 47, 3196 (2008).

[59] P. Varga and P. Török, The Gaussian Wave Solution of Maxwell's Equations and the Validity of Scalar Wave Approximation, Opt. Commun. 152, 108 (1998).

[60] The chiral coupling between spins $\mathbf{S}$ in a magnetic field $\mathbf{H}$ is driven by the interaction potential $-\mathbf{S} \cdot \mathbf{H}$, whereas molecular ratchets by a (one-dimensional $z$-) tilted washboard potential that typically writes as $A \cos [2 \pi z / \lambda]-F_{0} z$ where $A$ is the coupling constant, $\lambda$ is the washboard wavelength, and $F_{0}$ is the biasing external force. No such potentials can be derived in the chiral dissipative case, as we explain throughout our work.

[61] C. Jarzynski, Comparison of Far-from-Equilibrium Work Relations, C.R. Phys. 8, 495 (2007).

[62] A. Simon and A. Libchaber, Escape and Synchronization of a Brownian Particle, Phys. Rev. Lett. 68, 3375 (1992).

[63] G. Schnoering, L. V. Poulikakos, Y. Rosales-Cabara, A. Canaguier-Durand, D. J. Norris, and C. Genet, ThreeDimensional Enantiomeric Recognition of Optically Trapped Single Chiral Nanoparticles, Phys. Rev. Lett. 121, 023902 (2018).

[64] E. Vinegrad, U. Hananel, G. Markovich, and O. Cheshnovsky, Determination of Handedness in a Single 
Chiral Nanocrystal via Circularly Polarized Luminescence, ACS Nano 13, 601 (2019).

[65] P. Spaeth, S. Adhikari, L. Le, T. Jollans, S. Pud, W. Albrecht, T. Bauer, M. Caldarola, L. Kuipers, and M. Orrit, Circular Dichroism Measurement of Single Metal Nanoparticles Using Photothermal Imaging, Nano Lett. 19, 8934 (2019).

[66] J. Sachs, J.-P. Günther, A. G. Mark, and P. Fischer, Chiroptical Spectroscopy of a Freely Diffusing Single Nanoparticle, Nat. Commun. 11, 4513 (2020).

[67] P. Lodahl, S. Mahmoodian, S. Stobbe, A. Rauschenbeutel, P. Schneeweiss, J. Volz, H. Pichler, and P. Zoller, Chiral Quantum Optics, Nature (London) 541, 473 (2017).

[68] S. Mahmoodian, G. Calajó, D. E. Chang, K. Hammerer, and A. S. Sørensen, Dynamics of Many-Body Photon Bound
States in Chiral Waveguide QED, Phys. Rev. X 10, 031011 (2020).

[69] P. Talkner and P. Hänggi, Colloquium: Statistical Mechanics and Thermodynamics at Strong Coupling: Quantum and Classical, Rev. Mod. Phys. 92, 041002 (2020).

[70] C. Elouard, D. Herrera-Martí, M. Esposito, and A. Auffèves, Thermodynamics of Optical Bloch Equations, DOI: https:// doi.org/10.1088/1367-2630/abbd6e (2020).

[71] P. Hanggi, Escape from a Metastable State, J. Stat. Phys. 42, 105 (1986).

[72] P. E. Kloeden and E. Platen, Numerical Solutions of Stochastic Differential Equations, 1st ed. (Springer-Verlag, Berlin, 1992), Vol. 23.

[73] S. Schütz, S. B. Jäger, and G. Morigi, Thermodynamics and Dynamics of Atomic Self-Organization in an Optical Cavity, Phys. Rev. A 92, 063808 (2015). 